\title{
Mathematical modelling of avascular-tumour growth
}

\author{
J. P. WARD AND J. R. KING \\ Department of Theoretical Mechanics, University of Nottingham, Nottingham, \\ $N G 72 R D, U K$
}

\author{
[Received 8 March 1996 and in revised form 15 August 1996]
}

\begin{abstract}
A system of nonlinear partial differential equations is proposed as a model for the growth of an avascular-tumour spheroid. The model assumes a continuum of cells in two states, living or dead, and, depending on the concentration of a generic nutrient, the live cells may reproduce (expanding the tumour) or die (causing contraction). These volume changes resulting from cell birth and death generate a velocity field within the spheroid. Numerical solutions of the model reveal that after a period of time the variables settle to a constant profile propagating at a fixed speed. The travelling-wave limit is formulated and analytical solutions are found for a particular case. Numerical results for more general parameters compare well with these analytical solutions. Asymptotic techniques are applied to the physically relevant case of a small death rate, revealing two phases of growth retardation from the initial exponential growth, the first of which is due to nutrient-diffusion lımitations and the second to contraction during necrosis. In this limit, maximal and 'linear' phase growth speeds can be evaluated in terms of the model parameters.
\end{abstract}

Keywords: tumour growth; avascular; mathematical modelling; numerical solution; asymptotic analysis.

\section{Introduction}

Solid tumours arise from an alteration of a cell's genetic material causing it to respond differently to the host's growth regulators and this leads to the uncontrolled growth of these cells (Melicow, 1982). The early tumour cells, like the neighbouring normal cells, can obtain adequate nourishment (such as glucose and oxygen) from the existing vasculature, and hence the proliferation rate of these cells is regular, producing a growing spheroid of cells expanding at a near exponential rate. As the tumour grows the amount of nutrient that manages to diffuse to the centre decreases due to consumption by the outer cells and eventually the central cells become so deficient that their proliferative rate is reduced and cell division may indeed cease, with these cells becoming quiescent. These quiescent cells are still viable and they can recover on the restoration of sufficient nutrient (Freyer \& Schor, 1987). This reduction of proliferation within the spheroid retards its growth. As the tumour grows further, the continued absence of nutrients in the central regions will cause the cells there to die, forming a region of dead cells known as a necrotic core. As the spheroid continues to develop, the rim of adequately nourished viable cells at the surface becomes roughly constant in size, leading to a phase of near linear growth (Conger \& Ziskin, 1983; Freyer \& Sutherland, 1986). Eventually, after a period of a few weeks, the combined action of necrotic disintegration, accumulation of waste products (Vaupel et al., 1981), mitotic inhibitory factors (Freyer, 1988) and cell shedding (Landry et al., 1981) reduces the rate of 
growth of in vitro tumours, leading to a spheroid of a maximal (saturation) size (Inch $e t$ al., 1970; Folkman \& Hochberg, 1973; Haji-Karim \& Carlsson, 1978). At growth saturation there is still a relatively thin rim of proliferating cells, which reproduce at a rate that declines with depth into the tumour (Carlsson, 1977). However, tumours in vivo continue to develop by releasing agents known as tumour-angiogenesis factors (TAFs) which stimulate the growth of new capillaries from the neighbouring vasculature into the tumour mass, replenishing the supply of nutrients deeper in the tumour and allowing growth to continue to sizes well beyond the saturation level (Folkman, 1971). Beyond this level, malignant tumours may become invasive (metastatic), utilizing the body's cavities and vasculature to settle elsewhere, forming secondary growths in the host.

Mathematical models that relate the diffusion of nutrients and tumour heterogeneity were first proposed by Thomlinson \& Gray (1955) and then extended by Burton (1966) to link tumour growth with the size of the region in which the nutrient concentration is greater than some critical level. Greenspan (1972) later proposed a model which attempts to capture all the phases of avascular growth by adding the action of a mitotic inhibitor (produced within the tumour) and necrotic decomposition and by dividing the tumour into distinct compartments of proliferating, quiescent, and necrotic cells. This model thus incorporated the various phases of growth leading to saturation. A number of extensions to the Greenspan model have been investigated (Deakin, 1975; Maggelakis \& Adam, 1990; Maggelakis, 1992). A crucial feature of the Greenspan model is that the contraction rate is proportional to the size of the necrotic core; McElwain \& Morris (1978) showed that similar qualitative behaviour can be achieved by assuming that the only source of cell contraction is through apoptosis ('programmed' cell death, Moore, 1987) in the viable rim. Glass (1973) studied a single quasisteady reaction-diffusion equation for the inhibitor distribution in a tumour and its effects on eventual tumour saturation; this study has also spawned many subsequent investigations involving the effects of geometry and of different source functions representing tumour heterogeneity (Shymko \& Glass, 1976; McElwain \& Ponzo, 1977; Adam, 1986, 1987a,b; Adam \& Maggelakis, 1989; Chaplain \& Britton, 1993); however, Chaplain et al. (1994) showed that the same qualitative behaviour can be obtained from a spatially varying diffusion coefficient. More recently, Byrne \& Chaplain (1996) extended the assumptions of Greenspan to include the effects of apoptosis, and they generalized the assumptions on the inhibitor so that it could be viewed as the application of an anticancer drug. A very detailed model proposed by Casciari et al. (1992a) studies the evolution of the distributions of seven important metabolites within the spheroid, linking their concentrations with a simple growth equation to produce moderate agreement with experimental data. The effects of cell migration due to convection, diffusion, and chemotaxis (up nutrient gradients) were studied by McElwain \& Pettet (1993) to model experimental observations of the drifting of probes from the viable rim of spheroids to the necrotic core. Their approach generalized the growth- and nutrient-consumption terms of Greenspan; however, in contrast to the model presented in this paper, these terms were taken to be independent of the local live-cell density. These diffusion models all assume that the tumour is avascular and that the surface is the only source of nutrient; however, Byrne \& Chaplain (1995) included a source of nutrient within a non-necrotic tumour in order to model the growth of a vascularized tumour.

The model presented in this paper takes a rather different approach, although it again directly links growth with the local nutrient concentration. The model describes a continuum 
of live cells which, through local volume changes by cell growth and death, create movement described by a velocity field. The convective process within a spheroid during growth is thus considered. Previous models define cells to be dead when the nutrient concentration drops below some threshold; the current model differs from such models by assuming that cells die (and then contract) at a rate which depends on the local nutrient concentration; that is, cell death is a gradual process which does not occur instantaneously as a response to low-nutrient conditions. Note that, in contrast to previous diffusion models, no assumptions are made a priori about the tumour structure, the heterogeneity of the tumour being obtained from the solutions. The model is presented in Section 2 and, although it lacks mechanisms to cause growth saturation in its present form, the early exponential phase and the retardation to linear growth are demonstrated from the numerical simulations described in Section 3. In Section 4 the travelling-wave limit of the model is derived to capture the tumour's behaviour during the linear-growth phase, and analytical solutions are presented for a certain limiting case. Asymptotic analysis, based on a small-death-rate assumption, is carried out in Section 5, where, following on from an initial 'exponential' phase, two longer phases of growth retardation occur, the first of which is due to nutrient-diffusion limitations within the spheroid and the second to cellular contraction during necrosis through continued absence of nutrient in the core.

\section{Tumour-growth model}

\subsection{Mathematical model}

The aim of this study is to develop a deterministic tumour-growth model, capturing tumour heterogeneity without introducing distinct regions a priori, that is simple enough to be mathematically tractable. The tumour is viewed as an agglomeration of matter consisting of a continuum of cells in two states, living and dead. In the living state the matter is assumed to be able to expand (due to cell growth and division) at a rate which is dependent on the local availability of nutrient (treated as a single generic species). The irreversible transition from the live state to the dead state is assumed to cause a spontaneous volume loss, and its rate also depends on the local nutrient concentration. The local volume changes caused by these processes produces movement of the cells. The main quantities appearing in the model can be identified from this picture of tumour behaviour, namely, the distributions of the living cells and of the nutrient which fuels growth, together with the velocity field inside the tumour caused by the growth and death of cells.

We take $n(x, t)$ and $m(x, t)$ to be the living-and dead-cell concentrations, respectively, $v(x, t)$ to be the local velocity of cells, and $c(x, t)$ to be the nutrient concentration. The equations governing $n$ and $m$ are

$$
\begin{aligned}
\frac{\partial n}{\partial t}+\nabla \cdot(v n) & =\left[k_{\mathrm{m}}(c)-k_{\mathrm{d}}(c)\right] n, \\
\frac{\partial m}{\partial t}+\nabla \cdot(v m) & =k_{\mathrm{d}}(c) n,
\end{aligned}
$$

where $k_{\mathrm{m}}$ and $k_{\mathrm{d}}$ are the rates of cell mitosis and cell death, respectively, which are prescribed below. Equation (2.1) states that the rate of change of $n$ is determined by the difference in the rates of mitosis and death of the cells. The rate of change of $m$ must equal the death rate of cells, leading to equation (2.2). 
The rates of mitosis, $k_{\mathrm{m}}(c)$, and death, $k_{\mathrm{d}}(c)$, are assumed to be bounded and, respectively, increasing and decreasing functions of nutrient concentration, with $k_{\mathrm{m}}(0)=$ $0, k_{\mathrm{m}}(\infty)=A, k_{\mathrm{d}}(0)=B$, and $k_{\mathrm{d}}(\infty)=B(1-\sigma)$, where $A$ and $B$ are positive constants and $0 \leqslant \sigma \leqslant 1$. We adopt simple functional forms consistent with these assumptions; namely,

$$
\begin{aligned}
& k_{\mathrm{m}}(c)=\frac{A c^{m_{1}}}{c_{\mathrm{c}}^{m_{1}}+c^{m_{1}}}, \\
& k_{\mathrm{d}}(c)=B\left(1-\frac{\sigma c^{m_{2}}}{c_{\mathrm{d}}^{m_{2}}+c^{m_{2}}}\right),
\end{aligned}
$$

where the exponents $m_{1}, m_{2}>0$ govern the sharpness of change near the 'critical' concentrations $c_{\mathfrak{c}}$ and $c_{\mathrm{d}}$. Note that, even in optimal nutrient conditions, $k_{\mathrm{d}}>0$, implying that cell death still occurs, which reflects cell loss through apoptosis. These equations generalize the Michaelis-Menten kinetics $\left(m_{1}=m_{2}=1\right)$ which are often used to model cell kinetics (Lin, 1976; McElwain, 1978) and cellular nutrient consumption in tumours (Casciari $e t$ al., 1992b; Hlatky et al., 1988; Li, 1982a). The limits $m_{1}, m_{2} \rightarrow \infty$ reduce $k_{\mathrm{m}}$ and $k_{\mathrm{d}}$ to discontinuous step-function forms; namely,

$$
\begin{aligned}
& k_{\mathrm{m}}(c)=A \mathrm{H}\left(c-c_{\mathrm{c}}\right), \\
& k_{\mathrm{d}}(c)=B\left[1-\sigma \mathrm{H}\left(c-c_{d}\right)\right],
\end{aligned}
$$

where $\mathrm{H}(\cdot)$ is the Heaviside function; this will provide a useful simplification in future analysis.

We define $V_{\mathrm{L}}$ and $V_{\mathrm{D}}$ to be the mean volumes of a single living and dead cell, respectively; $V_{D}<V_{L}$ is assumed. It is assumed that the rate of volume change drives the movement of the cells, so the equation for the velocity $v$ is given by

$$
\nabla \cdot v=k_{\mathrm{m}}(c) n V_{\mathrm{L}}-k_{\mathrm{d}}(c) n\left(V_{\mathrm{L}}-V_{\mathrm{D}}\right)
$$

which states that the rate of volume change is given by the difference between the rates of volume increase, $k_{\mathrm{m}} n V_{\mathrm{L}}$, and decrease, $k_{\mathrm{d}} n\left(V_{\mathrm{L}}-V_{\mathrm{D}}\right)$, where $V_{\mathrm{L}}-V_{\mathrm{D}}$ is the spontaneous volume loss due to the death of a cell. It is assumed that the time scale of volume loss on cell death is much shorter than that of tumour growth.

The tumour is treated as being avascular, obtaining its nutrients only from the surrounding tissue or matrix through the tumour surface; the distribution of nutrient is governed by diffusion and consumption. It is assumed that Fick's laws model the diffusion of the nutrient into the interior of the tumour, with the diffusion coefficient $D$ taken to be constant since spheroid heterogeneity does not significantly affect diffusion rates (Casciari et al., 1988). It is assumed that the rate at which the nutrient is consumed is governed by two terms, the first represents nutrient consumption by normal (that is, nonmitotic) processes, $\gamma(c) n$, while the second describes the additional amount consumed during mitosis and is given by $\beta k_{\mathrm{m}}(c) n$. Combining these assumptions yields the following equation for $c$ :

$$
\frac{\partial c}{\partial t}+\nabla \cdot(v c)=\nabla \cdot(D \nabla c)-\left[\beta k_{\mathrm{m}}(c)+\gamma(c)\right] n,
$$

provided that $c$ remains non-negative. It has been reported in several studies that oxygen 
(Mueller-Klieser \& Sutherland, 1982; Mueller-Klieser et al., 1985; Hlatky et al., 1988) and glucose (Li, 1982a; Mueller-Klieser et al., 1983) concentrations in the interior of larger spheroids can be negligible. Equation (2.7) may predict that $c$ becomes negative if $\gamma(c)$ is, for example, nonvanishing as $c \rightarrow 0$. If this possibility arises we must further impose continuity of $c$ and its nomal derivative at a moving boundary (say $\Gamma(t)$ ) enclosing a region with zero concentration, so that on $\Gamma(t)$ we have $c=\nabla c \cdot \hat{n}=0$.

Since $m$ does not occur in equations (2.1), (2.6), and (2.7), it is decoupled from the other variables. We assume there are no voids, so it follows that $m$ is given by

$$
V_{\mathrm{L}} n(x, t)+V_{\mathrm{D}} m(x, t)=1 ;
$$

this can also be derived from (2.1), (2.2), and (2.6).

We will henceforth exploit the spherical symmetry of the problem and hence, in particular, we avoid the need to prescribe constitutive equations to determine $v$. This simplification implies that the three equations (2.1), (2.6), and (2.7) are adequate to determine $n, c$, and the radial velocity $v$ as functions of the independent variables $r=|x|$ and $t$.

To complete the above system we must impose appropriate initial and boundary conditions. We shall take the initial state of the tumour to be a single mutated cell whose radius $S(0)$ is given by (2.9), $S(t)$ being the spheroid radius as a function of time and thus it is the coordinate of a moving boundary. While a continuum approach cannot then be expected to be valid for very small times, it rapidly becomes acceptable as cell multiplication produces a significant number of cells. The following initial conditions are therefore adopted:

$$
S(0)=\left(3 V_{\mathrm{L}} / 4 \pi\right)^{\frac{1}{3}}, \quad n(r, 0)=1 / V_{\mathrm{L}}, \quad c(r, 0)=c_{0},
$$

where the constant $c_{0}$ is the external nutrient concentration.

As boundary conditions we impose:

$$
\begin{array}{cc}
\frac{\mathrm{d} S}{\mathrm{~d} t}=v(S(t), t), & c(S(t), t)=c_{0}, \\
\frac{\partial c(0, t)}{\partial r}=0, & v(0, t)=0 .
\end{array}
$$

where the first boundary condition of (2.10) states that the spheroid boundary moves with the local velocity.

\subsection{Non-dimensionalization}

Denoting dimensionless variables by carets, we introduce the following rescalings which are based on the initial conditions:

$$
\begin{gathered}
n=\hat{n} / V_{\mathrm{L}}, \quad c=c_{0} \hat{c}, \\
t=\hat{t} / A, \quad r=r_{0} \hat{r}, \quad S=r_{0} \hat{S},
\end{gathered}
$$

where $r_{0}=S(0)=\left(3 V_{L} / 4 \pi\right)^{1 / 3}$. We thus obtain the following system

$$
\frac{\partial \hat{n}}{\partial \hat{t}}+\hat{v} \frac{\partial \hat{n}}{\partial \hat{r}}=[a(\hat{c})-b(\hat{c}) \hat{n}] \hat{n}
$$




$$
\begin{aligned}
v\left(\frac{\partial \hat{c}}{\partial \hat{t}}+\hat{v} \frac{\partial \hat{c}}{\partial \hat{r}}+b(\hat{c}) \hat{c} \hat{n}\right) & =\frac{1}{\hat{r}^{2}} \frac{\partial}{\partial \hat{r}}\left(\hat{r}^{2} \frac{\partial \hat{c}}{\partial \hat{r}}\right)-k(\hat{c}) \hat{n}, \\
\frac{1}{\hat{r}^{2}} \frac{\partial\left(\hat{r}^{2} \hat{v}\right)}{\partial \hat{r}} & =b(\hat{c}) \hat{n},
\end{aligned}
$$

where the reaction terms $a(\hat{c}), b(\hat{c})$, and $k(\hat{c})$ are given in terms of the dimensionless quantities

$$
\hat{k}_{\mathrm{m}}(\hat{c})=\frac{\hat{c}^{m_{1}}}{\hat{c}^{m_{1}}+\hat{c}_{\mathrm{c}}^{m_{1}}}, \quad \hat{k}_{\mathrm{d}}(\hat{c})=\frac{B}{A}\left(1-\sigma \frac{\hat{c}^{m_{2}}}{\hat{c}^{m_{2}}+\hat{c}_{\mathrm{d}}^{m_{2}}}\right),
$$

where $\hat{c}_{\mathrm{c}}=c_{\mathrm{c}} / c_{0}$ and $\hat{c}_{\mathrm{d}}=c_{\mathrm{d}} / c_{0}$, by the expressions

$$
\begin{aligned}
& a(\hat{c})=\hat{k}_{\mathrm{m}}(\hat{c})-\hat{k}_{\mathrm{d}}(\hat{c}), \\
& b(\hat{c})=\hat{k}_{\mathrm{m}}(\hat{c})-(1-\delta) \hat{k}_{\mathrm{d}}(\hat{c})=a(\hat{c})+\delta \hat{k}_{\mathrm{d}}(\hat{c}), \\
& k(\hat{c})=\hat{\beta} \hat{k}_{\mathrm{m}}(\hat{c})+\hat{\gamma}(\hat{c}),
\end{aligned}
$$

and where $\delta=V_{\mathrm{D}} / V_{\mathrm{L}} \in[0,1], \hat{\beta}=r_{0}^{2} \beta A / D V_{\mathrm{L}} c_{0}, \hat{\gamma}(c)=r_{0}^{2} \gamma(c) / D V_{\mathrm{L}} c_{0}$, and $v=$ $A r_{0}^{2} / D$. The function $a(\hat{c})$ represents the rate of population growth, $b(\hat{c})$ represents the rate of volume change, and $k(\hat{c})$ is proportional to the rate of consumption of nutrient. Note that these three functions are monotonically increasing in $\hat{c}$ and, since $\hat{k}_{\mathrm{m}}, \hat{k}_{\mathrm{d}} \geqslant 0$, we have $a(\hat{c}) \leqslant b(\hat{c})$. In what follows the carets are dropped from the variables and parameters; however, in the Appendix quantities without carets revert to being dimensional quantities.

Using the data given in the Appendix, the time scale 1/ $A$ is about 14 hours and we have $v \approx 10^{-5}$; henceforth we therefore replace $(2.13)$ by the quasisteady approximation

$$
\frac{1}{r^{2}} \frac{\partial}{\partial r}\left(r^{2} \frac{\partial c}{\partial r}\right)=k(c) n
$$

It is unlikely that the nutrient-consumption rate is directly proportional to the rate at which mitosis occurs, hence the inclusion of $\gamma(c)$ in $k(c)$. Nevertheless, as described in the Appendix, experimental results suggest that it suffices to take $\gamma(c) \equiv 0$, and we shall henceforth adopt this assumption.

To complete this system the dimensionless initial and boundary conditions are

$$
\begin{gathered}
n(r, 0)=1, \quad S(0)=1, \\
c(S(t), t)=1, \quad \frac{\mathrm{d} S}{\mathrm{~d} t}=v(S(t), t), \quad \frac{\partial c(0, t)}{\partial r}=0, \quad v(0, t)=0 .
\end{gathered}
$$

The system thus consists of the first-order partial differential equation (2.12), the secondorder (quasisteady) differential equation (2.18), and the first-order equation (2.14), defined on a domain $r<S(t)$ with an unknown moving boundary $S(t)$. It is worth noting that we may easily solve for $n(S(t), t)$ ), giving

$$
n(S(t), t)=\frac{a(1) \mathrm{e}^{a(1) t}}{a(1)-b(1)\left(1-\mathrm{e}^{a(1) t}\right)} ;
$$

this gives an upper bound on $n(r, t)$. 


\section{Numerical solution}

\subsection{Numerical methods}

The system $(2.12,2.14,2.18-2.20)$ is solved numerically after first fixing the moving boundary by writing $r=S(t) r^{\dagger}$, thus mapping the tumour onto the unit interval. The equations are solved sequentially using finite-difference methods in a predictor-corrector scheme: first, $S(t)$ is updated using the trapezium method; equation (2.18) is approximated using the National Algorithms Group (NAG) routine D02RAF, which uses a finitedifference approach; the trapezium method is also used to approximate equation (2.14); finally, an implicit, second-order accurate extension to the scheme proposed by Courant et al. (1952, §1), is adopted for approximating (2.12). This sequence is then repeated on the correction loops. For large $S$ the rescaling has the drawback (for a uniform mesh) of condensing the regions of most variation into a boundary layer of $O\left(S^{-1}\right)$. This is partly overcome by the use of a contracting mesh, where successive spatial increments are determined by $\Delta r_{j+1}^{\dagger}=\lambda \Delta r_{j}^{\dagger}$, where $\Delta r_{j}^{\dagger}$ is the size of the $j$ th increment and $\lambda$ is a constant factor less than one.

\subsection{Numerical results}

Figure 1 shows the spheroid radius as a function of time and Figs. 2-4 show the distributions of the dependent variables $n, c$, and $v$, respectively, at equal time intervals, for the physically motivated parameter values (see the Appendix) $B / A=1, \sigma=0.9, \delta=$ $0.5, \beta=0.005, c_{\mathrm{c}}=0.1, c_{\mathrm{d}}=0.05, m_{1}=m_{2}=1$. Observe that, after an initial period in which the rate of growth increases in Fig. 1 (roughly $t<10$ ), the growth slows down, ultimately becoming linear. A close inspection of the curve for $15<t<20$, say, reveals

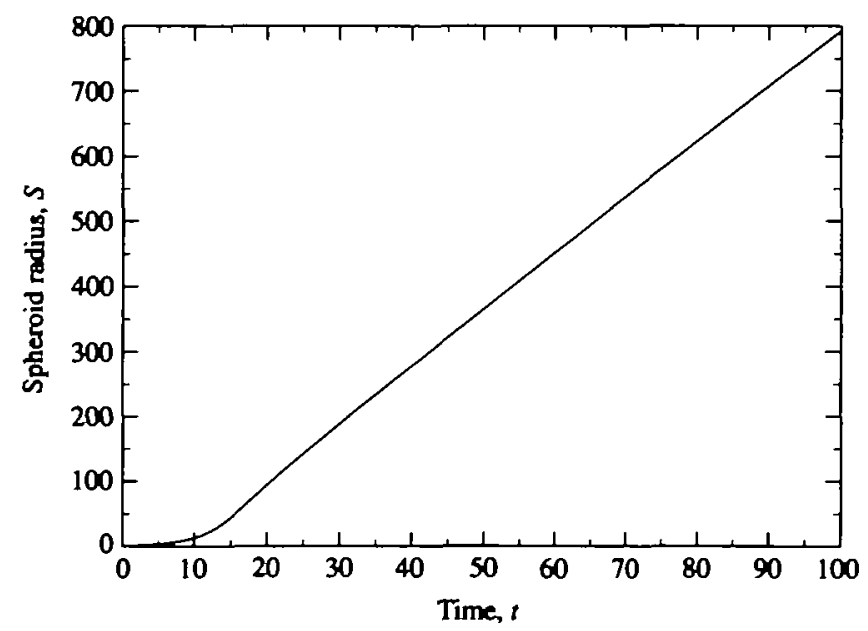

FIG. 1. The dimensionless tumour radius, $S(t)$, ploted against time. In dimensional terms $t=1$ typically represents 14 hours and the speed of linear growth is approximately $2 \mu \mathrm{m} \mathrm{h}^{-1}$. 


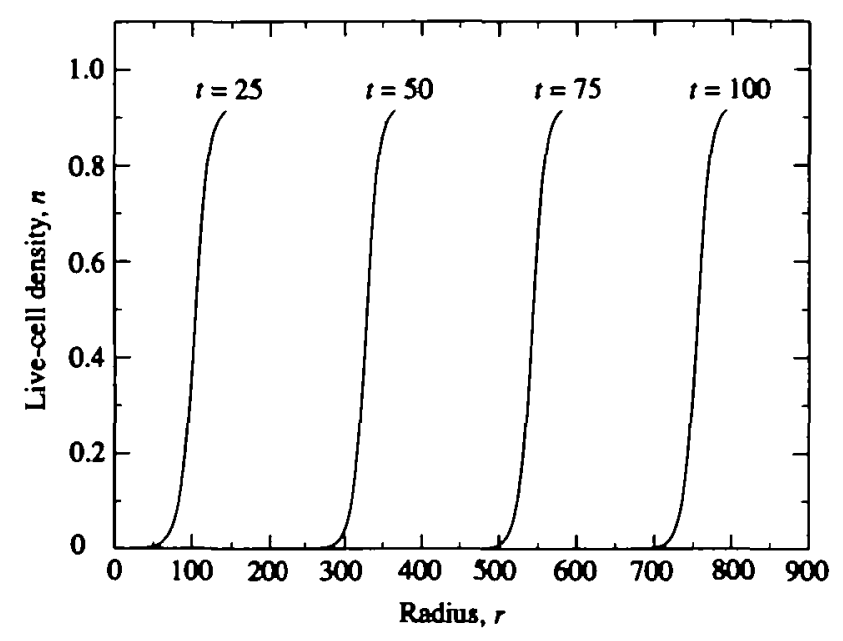

FIG. 2. The spatial distnbution of the live-cell density for four values of $t$.

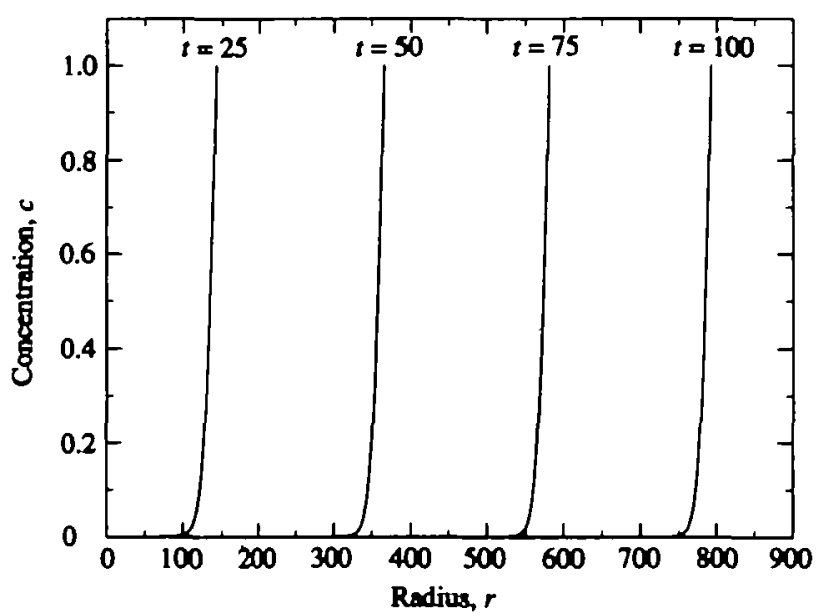

FJG. 3. The nutrient concentration plotted against radius for four values of $t$.

a slight kink as the growth rate decelerates a little before reaching the linear phase. This behaviour is predicted in the analysis of Section 5 and is due to the time delay from when cells become quiescent to when they die; this feature does not seem to have been predicted in previous studies. The live-cell density (Fig. 2) is relatively constant in a small region beneath the cell surface, dropping sharply towards zero deeper into the tumour, reflecting a well-defined viable rim and a necrotic core. It should be stressed that such regions arise 


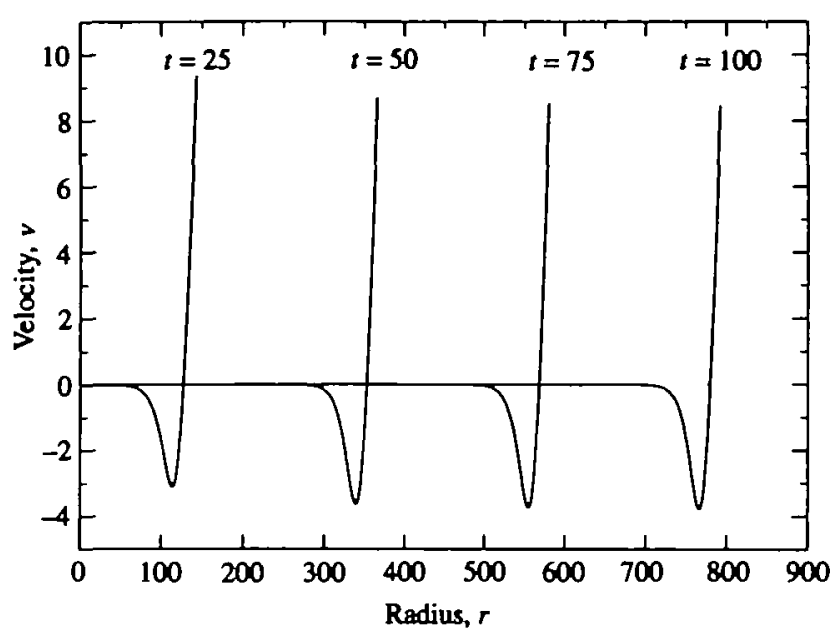

FIG 4. The velocity plotted against radius for four values of $t$.

naturally from the model rather than being assumed a priori. The nutrient concentration (Fig. 3) decreases sharply through the viable rim and tends to a constant level in the core $\left(O\left(10^{-3}\right)\right.$ in this case), due to the nearly complete necrosis $(n \approx 0)$ in this region. Although the cells at the surface are adequately nourished, the slight decline in cell density there reflects loss by apoptosis. The velocity within the tumour (Fig. 4) decreases very rapidly from a positive value towards a negative minimum, before approaching zero in the necrotic core. The region of negative velocity reflects the fact that volume loss by cell death is greater there than the volume gain through mitosis. The initial exponential increase in the cell population and the eventual retardation is depicted in Fig. 5. The growth falls away from the exponential phase when the spheroid consists of $O\left(10^{5}\right)$ cells; this is consistent with experimental observations (for example, Koch et al., 1973; Freyer \& Sutherland, 1986) in fairly generous nutrient conditions.

The sources for much of the data used for this simulation are indicated in the Appendix, the remainder being 'best' estimates. Quantitatively, the simulation predicts a growth speed during the linear phase of around $2 \mu \mathrm{m} \mathrm{h}^{-1}$, which is consistent with the reported speeds of approximately 1-3 $\mu \mathrm{m} \mathrm{h}^{-1}$ measured by Conger \& Ziskin (1983) and of $1 \mu \mathrm{m} \mathrm{h}^{-1}$ measured by $\mathrm{Li}(1982 \mathrm{~b}$ ). Further, the width of the viable rim (say where $n>0.5$ ) is equivalent to about 20 cells, which overestimates some reported widths. In terms of the model, these features depend crucially on the size of function $k(c)$ and on the external nutrient concentration. For example, setting $\beta=0.01, c_{\mathrm{c}}=0.2$, and $c_{\mathrm{d}}=0.1$ instead leads to a growth speed of about $1.1 \mathrm{\mu m} \mathrm{h}^{-1}$ and to a viable-rim thickness of about 10 cells; this is more consistent with the reported experimental results. 


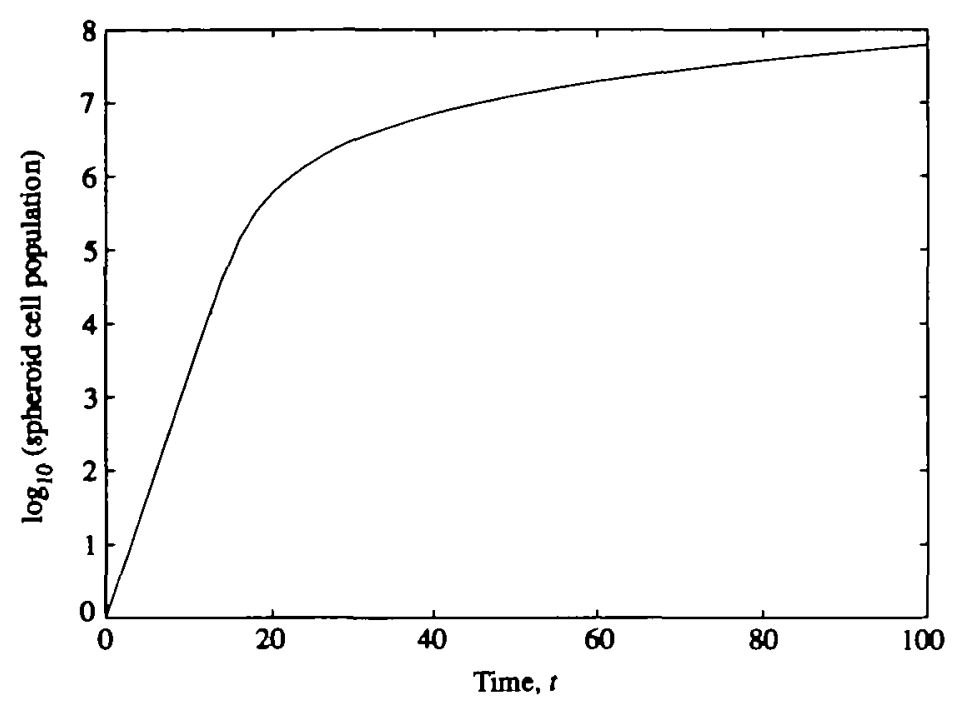

FIG. 5. A plot of $\log _{10}$ (spheroid cell population) against time

\section{Large-time behaviour}

\subsection{Formulation of travelling-wave equations}

The numerical results suggest that after the initial transient behaviour the tumour growth becomes nearly linear, with the profiles maintaining a fixed shape while propagating outwards. This type of behaviour is consistent with experimental observations in the intermediate phase of spheroid growth. In order to understand the behaviour of the model during this phase we now consider the travelling-wave limit of the model equations (2.12-2.14). We assume that at large times the tumour grows with a constant speed $U$ (which remains to be determined), so $S(t) \sim U t$, and we introduce a travelling-wave coordinate $z=r-S(t)$, with $z<0$. The following system of ordinary differential equations then represents the leading-order balance in the viable rim as $t \rightarrow \infty$ :

$$
\begin{aligned}
(v-U) n^{\prime} & =[a(c)-b(c) n] n, \\
c^{\prime \prime} & =k(c) n, \\
v^{\prime} & =b(c) n,
\end{aligned}
$$

where the primes denote differentiation with respect to $z$; the terms in $r^{-1} \partial / \partial r$ are $O\left(S^{-1}\right)$ as $S \rightarrow \infty$ and are thus negligible as $t \rightarrow \infty$.

This system is subject to the following boundary conditions:

$$
n(0)=a(1) / b(1), \quad c(0)=1, \quad v(0)=U, \quad c^{\prime}(-\infty)=0, \quad v(-\infty)=0,
$$

which all follow from $(2.20)$ and $(2.21)$ as $t \rightarrow \infty$. The first of these boundary conditions is equivalent to the requirement that $n^{\prime}(0)$ be bounded.

Observe that we are left with a fourth-order system with five boundary conditions; this system is sufficient to determine the unknown wave speed $U$ and the four variables $n, c, c^{\prime}$, 
and $v$. Using (4.3) together with $[(v-U) n]^{\prime}=a(c) n$ we obtain the following integral equation for $n$ :

$$
n(z)=\frac{\int_{z}^{0} a(c(\psi)) n(\psi) \mathrm{d} \psi}{\int_{z}^{0} b(c(\psi)) n(\psi) \mathrm{d} \psi}
$$

\subsection{Analytical solutions for step-function forms of $k_{\mathrm{m}}$ and $k_{\mathrm{d}}$}

Analytical solutions to $(4.2-4.5)$ can be found in the step-function limits for mitotic and death-rate functions, corresponding to taking $m_{1}, m_{2} \rightarrow \infty$, with

$$
\begin{aligned}
& a(c)=H\left(c-c_{\mathrm{c}}\right)-B\left[1-\sigma H\left(c-c_{d}\right)\right] / A, \\
& b(c)=a(c)+\delta B\left[1-\sigma H\left(c-c_{\mathrm{d}}\right)\right] / A, \\
& k(c)=\beta H\left(c-c_{\mathrm{c}}\right) .
\end{aligned}
$$

Under these assumptions further consumption of the nutrient ceases where $c$ drops to $c_{\mathfrak{c}}$, which occurs at an a priori unknown moving boundary. The cessation of consumption when $c=c_{c}$ implies that if $c_{d} \leqslant c_{c}$ there can only be one moving boundary, whilst if $c_{d}>c_{c}$ there are two moving boundaries corresponding to $c=c_{\mathrm{d}}$ and $c=c_{\mathrm{c}}$. For simplicity we shall assume that $c_{\mathrm{c}} \geqslant c_{\mathrm{d}}$, in which case there are two distinct regions to consider corresponding to where $c>c_{\mathrm{c}}$ and where $c \equiv c_{\mathrm{c}}$. We also assume $\delta>0$ and $k_{\mathrm{d}} \not \equiv 0$ and use the notation

$$
\begin{aligned}
& c>c_{\mathrm{c}} \Rightarrow a(c)=a^{+}>0, b(c)=b^{+}>0, k(c)=\beta, \\
& c \leqslant c_{\mathrm{c}} \Rightarrow a(c)=-a^{-}<0, b(c)=-b^{-}<0, k(c)=0 .
\end{aligned}
$$

Since $a(c)<b(c)$ for $\delta>0$ we have $a^{+} b^{-} / a^{-} b^{+}<1$. Equation (4.2) implies that $c^{\prime \prime} \geqslant 0$ and, since $c^{\prime}(-\infty)=0, c$ is increasing in $z$. There is thus at most one point, $z=Z$ say, at which $c$ increases away from $c_{\mathrm{c}}$. We now give the solution to the system in (i) $z>Z$ and (ii) $z<Z$, imposing continuity of $n, c, c^{\prime}$, and $v$ at $z=Z$.

(i) $z>Z$. Using (4.3) and (4.5), and integrating (4.2), the solutions for the region $z>Z$, where $c>c_{\mathrm{c}}$, are

$$
\begin{aligned}
n & =\frac{a^{+}}{b^{+}}, \\
c & =1+\frac{\beta a^{+}}{2 b^{+}} z(z-Z)-\frac{z}{Z}\left(1-c_{\mathrm{c}}\right), \\
v & =U+a^{+} z,
\end{aligned}
$$

since $n(0)=a^{+} / b^{+}, v(0)=U, c(0)=1$, and $c(Z)=c_{c} ;$ and by imposing $c^{\prime}(Z)=$ 0 we can deduce that

$$
Z=-\left(2 \frac{b^{+}}{a^{+}} \frac{\left(1-c_{c}\right)}{\beta}\right)^{\frac{1}{2}}
$$


(ii) $z<Z$. For the region $z \leqslant Z$, where $c \equiv c_{\mathrm{c}}$, it is convenient in the analysis to introduce the function $N(z)=\int_{z}^{Z} n(\psi) \mathrm{d} \psi$, so from (4.5) we have

$$
n=-\frac{\mathrm{d} N}{\mathrm{~d} z}=\frac{a^{-}}{b^{-}} \frac{N^{*}-N}{N^{\ddagger}-N}
$$

where $N^{*}=-\left(a^{+2} / b^{+} a^{-}\right) Z>0$ and $N^{\ddagger}=-\left(a^{+} / b^{-}\right) Z$, so that $N^{*}<N^{\ddagger}$. Note from (4.10) that $N=N^{*}$ is the only stationary point of (4.10); this point is linearly stable as $z \rightarrow-\infty$, so we can deduce that $n(-\infty)=0$, implying necrosis. Solving for $N$ and $v$ we get

$$
\begin{aligned}
-\frac{a^{-}}{b^{-}}(Z-z) & =-N+\left(N^{\ddagger}-N^{*}\right) \ln \left(\frac{N^{*}-N}{N^{*}}\right), \\
v & =U+a^{+} Z+b^{-} N .
\end{aligned}
$$

Imposing $v(-\infty)=0$ and continuity at $z=Z$ we therefore finally obtain the growth speed

$$
U=-a^{+} Z\left(1-\frac{N^{*}}{N^{\ddagger}}\right)=\delta\left[2 \frac{\left(1-c_{\mathrm{c}}\right)}{\beta} \frac{a^{+}}{b^{+}}\right]^{\frac{1}{2}}
$$

this gives a unique speed $U$. The distribution of live cells for $n<a^{+} / b^{+}$can be calculated from equations (4.10) and (4.11), and in particular for $n \ll 1$ we have

$$
Z-z \sim-\frac{U}{B / A} \ln (n)
$$

which gives an indication of the depth to which quiescent cells survive within the spheroid.

If we set $B / A=0$ and $\delta=1$, so that $a^{+} / b^{+}=1$, then $U=-Z=\left[2\left(1-c_{\mathrm{c}}\right) / \beta\right]^{\frac{1}{2}}$, giving a solution identical to that of Greenspan (1972) in the case of no inhibitory effects or necrotic contraction ( $\gamma=P=0$ in his model); this is to be expected since, in this case, the models are equivalent.

A similar analysis can be performed for the case $c_{\mathrm{c}}<c_{\mathrm{d}}$, which is complicated by the presence of two moving boundaries, but the details will not be pursued here.

\subsection{Numerical solution of travelling-wave equations}

For general values of $m_{1}$ and $m_{2}$ we must resort to numerical methods for solution of the travelling-wave ordinary differential equations. Far-field analysis for $z \rightarrow-\infty$ reveals, provided $\delta>0$ and neither $k_{\mathrm{m}}(c)$ nor $k_{\mathrm{d}}(c)$ is identically zero, that $n \rightarrow 0, v \rightarrow 0$, and $c \rightarrow C_{0}$ (a constant). Linearizing the system $(4.1-4.3)$ about these values we can derive a set of asymptotic representations of the solutions for $-z \gg 1$, and for numerical purposes this allows us to truncate to a finite domain on which we solve the relevant twopoint boundary-value problem. If we take $L$ to be the length of the truncated domain, the 
equations are rescaled to the unit interval using $y=z / L+1$ and, defining $g=\mathrm{d} c / \mathrm{d} z$, the following system is to be solved numerically:

$$
\begin{aligned}
n^{\prime} & =L \frac{n[a(c)-b(c) n]}{v-U}, \\
c^{\prime} & =L g \\
g^{\prime} & =L k(c) n, \\
v^{\prime} & =L b(c) n,
\end{aligned}
$$

where the primes now denote differentiation with respect to $y$. This system is subject to the boundary conditions

$$
\begin{array}{ll}
n(0)=N_{0} \exp \left(\frac{a\left(C_{0}\right) L}{U}\right), & n(1)=\frac{a(1)}{b(1)}, \\
c(0)=C_{0}+\left(\frac{U}{a\left(C_{0}\right)}\right)^{2} k\left(C_{0}\right) n(0), & c(1)=1, \\
g(0)=-\frac{a\left(C_{0}\right)}{U}\left[c(0)-C_{0}\right], & v(1)=U, \\
v(0)=-\frac{b\left(C_{0}\right)}{a\left(C_{0}\right)} U n(0), &
\end{array}
$$

where the constants $N_{0}$ and $C_{0}$ must be determined as part of the solution. For computational purposes we also require the value of $n^{\prime}(1)$, which is obtained by applying

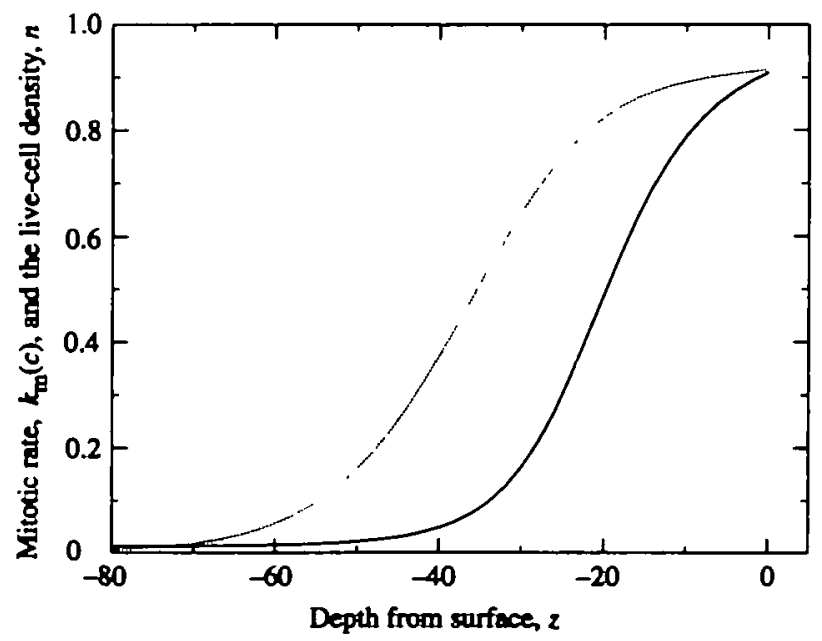

FIG. 6. (-) The mitotic rate and (..) the live-cell density near the surface of the spheroid during the lineargrowth phase. 


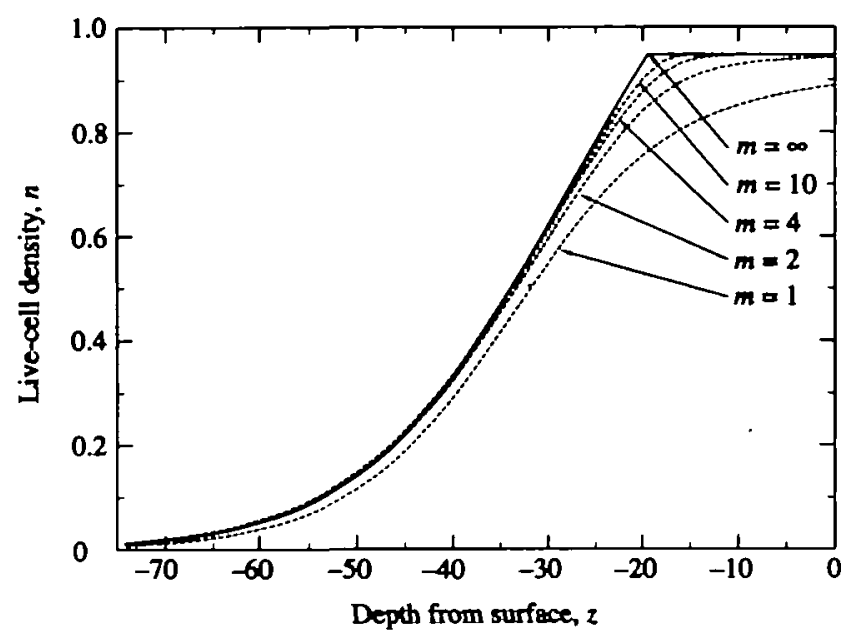

FIG 7. Plots of the live-cell density against the tumour depth for five values of the exponent $m=m_{1}=m_{2}$.

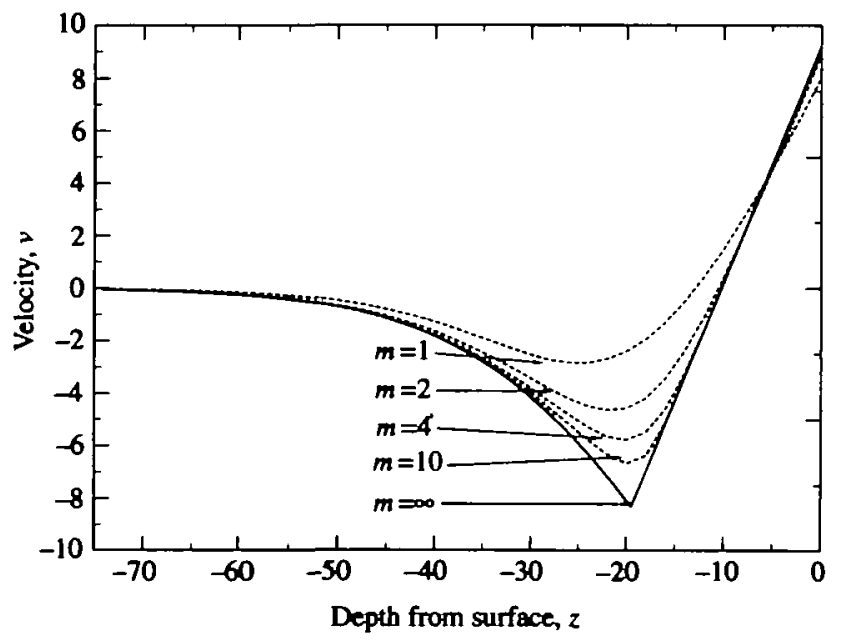

FIG. 8. Plors of the internal velocity against the tumour depth for five values of the exponent $m=m_{1}=m_{2}$.

L'Hôpital's rule to (4.15), as

$$
n^{\prime}(1)=L \frac{g(1)}{2 b(1)}\left(\left.\frac{\mathrm{d} a}{\mathrm{~d} c}\right|_{c=1}-\left.\frac{a(1)}{b(1)} \frac{\mathrm{d} b}{\mathrm{~d} c}\right|_{c=1}\right)
$$


By fixing $L$, we have a fourth-order system of ordinary differential equations with three parameters $\left(U, N_{0}, C_{0}\right)$ to be determined. The NAG routine D02AGF, which employs a shooting and matching technique with Newton's iteration, was used to solve this system.

A close-up of the viable rim is given in Fig. 6, where the mitotic rate is plotted together with the live-cell density for the set of parameters given in Section 3.2. Figure 6 illustrates the decline of the proliferative properties of cells with depth into the tumour, producing a region of nearly quiescent cells; these features are consistent with experimental observation (Freyer \& Schor, 1987), and again they were not an a priori imposition on the model. Figures 7 and 8 show the distributions from the tumour surface of $n$ and $v$, respectively, for $B / A=1, \sigma=0.9, \beta=0.005, c_{c}=c_{d}=0.1$ and for five values of the mitoticand death-rate exponents $\left(m_{1}=m_{2}=m\right)$, so that the relevance of the analytical solutions (for $m=\infty$ ) given in Section 4.2 can be examined for more general values of $k_{\mathrm{m}}$ and $k_{\mathrm{d}}$. Figure 7 shows that the solutions for $m \geqslant 4$ are reasonably close to the analytical solutions; and for $m=10$, say, most of the plateau of the $n$-distribution for $z>Z$ is maintained. However, the analytical solution is a poor approximation for $m=1$. The variation in $m$ has a significant effect on the velocity, especially on its minimum, although the wave speed $U$ predicted by the analysis of Section 4.2 gives a reasonable approximation for $m \geqslant 2$.

The effects of the death-rate parameter $B / A$ on the viable-rim size and the linear-phasegrowth velocity are shown in Figs. 9 and 10 (the other parameter values are those given above). In Fig. 9 a measure of the thickness of the viable rim (arbitrarily defined to be the region in which $n>0.1$ ) is plotted against $B / A$. Increasing $B / A$ implies that the rate of cell death is increased in the nutrient-deficient region in the trailing part of the rim, so that the width of the rim might be expected to be reduced; however, for the higher values of $m$ the width of the rim increases again for large $B / A$; this is due to the surface cell density being so low that the consequent reduced rate of nutrient consumption allows sufficient nutrient to penetrate deeper into the tumour. The curves drop back to zero as $n(0)$

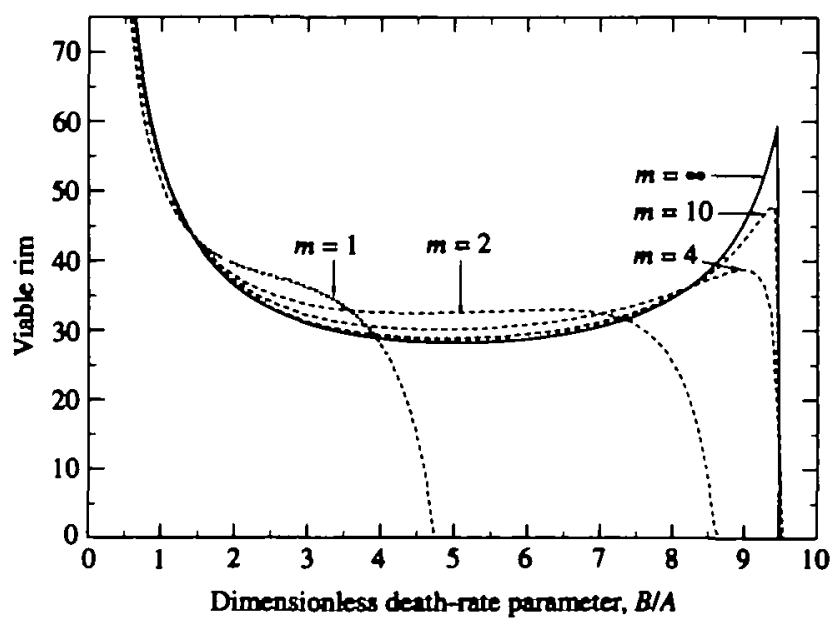

FIG 9. The effect of the death-rate parameter on a measure of the thickness of the viable rim. 


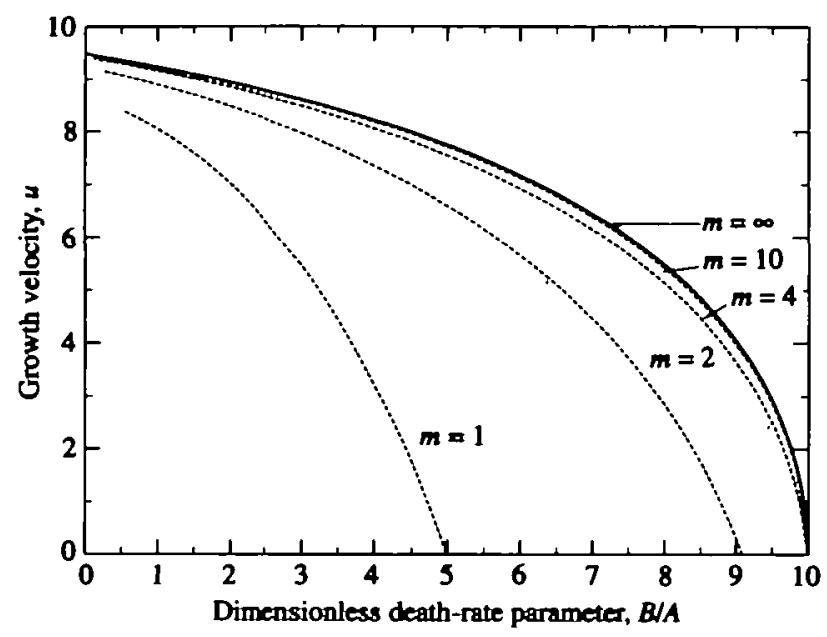

FIG. 10. The effect of the dimensinless death-rate parameter on the linear-phase-growth velocity.

approaches 0.1 . The expected behaviour of a reduction in the growth velocity on increasing $B / A$ is demonstrated in Fig. 10 . The point where $U=0$ corresponds to the value of $B / A$ being such that $a(1)=0$; clearly, if $a(1) \leqslant 0$ there is no region in the spheroid where the mitotic rate exceeds the death rate, implying that the tumour will die out.

\section{The slow-death-rate limit, $B / A \ll 1$}

\subsection{Introduction}

In a nutrient-rich environment a small number of cells die through apoptosis and, if we take $\theta$ to be the proportion of dead cells, we can derive the relationship $B / A=\theta /(1-\sigma)$ (see the Appendix). In such circumstances it is known that only a small proportion of cells die, suggesting $\theta \ll 1$, and if we assume that $1-\sigma=O(1)$ then the dimensionless quantity $B / A=\varepsilon \ll 1$ and the death rate $k_{\mathrm{d}}$ is $O(\varepsilon)$. Defining $\bar{k}_{\mathrm{d}}(c)$ by $k_{\mathrm{d}}(c)=\varepsilon \bar{k}_{\mathrm{d}}(c)$, the system of equations is

$$
\begin{aligned}
\frac{\partial n}{\partial t}+v \frac{\partial n}{\partial r} & =k_{\mathrm{m}}(c) n(1-n)-\varepsilon \bar{k}_{\mathrm{d}}(c) n[1-(1-\delta) n], \\
\frac{1}{r^{2}} \frac{\partial}{\partial r}\left(r^{2} \frac{\partial c}{\partial r}\right) & =\beta k_{\mathrm{m}}(c) n, \\
\frac{1}{r^{2}} \frac{\partial\left(r^{2} v\right)}{\partial r} & =k_{\mathrm{m}}(c) n-\varepsilon(1-\delta) \bar{k}_{\mathrm{d}}(c) n,
\end{aligned}
$$

which we shall now analyse in the limit $\varepsilon \rightarrow 0$. The initial and boundary conditions are as before.

Assuming a regular expansion of $n$ of the form $n \sim n_{0}+\varepsilon n_{1}$, it is easily demonstrated that positivity of $k_{\mathrm{m}}$ and $\bar{k}_{\mathrm{d}}$ implies $n_{0}=1$ and $n_{1} \geqslant-\delta t$, where the latter suggests that 
the minimum time scale for $1-n=O(1)$ is $t=O(1 / \varepsilon)$. This leads us to consider two main time scales for tumour development, the first being $t=O(1)$, for which $n \sim 1$, and the second being $t=O(1 / \varepsilon)$, when necrosis begins. The analysis will demonstrate that, following an initial near-exponential-growth phase, retardation occurs towards two successive asymptotic speeds: the first is due to nutrient-diffusion limitations predicting a maximum growth speed for the spheroid, while on a longer time scale further retardation occurs due to cell contraction $(\delta<1)$ during necrosis, leading to the second (slower) growth speed.

\section{$5.2 t=O(1)$}

5.2.1 Formulation. Since $n \sim 1$ holds on this time scale for all $r$, the leading-order governing equations are

$$
\begin{aligned}
\frac{1}{r^{2}} \frac{\partial}{\partial r}\left(r^{2} \frac{\partial c}{\partial r}\right) & =\beta \frac{c^{m_{1}}}{c_{\mathrm{c}}^{m_{1}}+c^{m_{1}}} \\
v & =\frac{1}{\beta} \frac{\partial c}{\partial r}
\end{aligned}
$$

note that we have suppressed the conventional suffix zero here in denoting the leadingorder solution since it will be needed in a subsidiary expansion below.

On this time scale the leading-order system is a moving-boundary problem for the single equation (5.1), subject to

$$
\frac{\partial c(0, t)}{\partial r}=0, \quad c(S, t)=1, \quad \frac{\mathrm{d} S}{\mathrm{~d} t}=\frac{1}{\beta} \frac{\partial c(S, t)}{\partial r}, \quad S(0)=1 .
$$

5.2.2 Small- $\beta$ behaviour. The experimental data given in the Appendix suggest that $\beta$ lies in the range $0.005-0.1$, which prompts us to study the further limit in which $\beta \rightarrow 0$ in (5.1-5.3). Expanding in powers of $\beta$ we find that for $t=O(1)$

$$
\begin{aligned}
& c \sim 1-\beta \frac{k_{m}(1)}{6}\left(S_{0}^{2}-r^{2}\right)-\beta^{2} \frac{k_{m}(1)}{3}\left(S_{0} S_{1}-\frac{k_{m}^{\prime}(1)}{120}\left(7 S_{0}^{2}-3 r^{2}\right)\left(S_{0}^{2}-r^{2}\right)\right), \\
& v \sim \frac{k_{\mathrm{m}}(1)}{3} r-\beta \frac{k_{\mathrm{m}}(1) k_{\mathrm{m}}^{\prime}(1)}{90} r\left(5 S_{0}^{2}-3 r^{2}\right), \\
& S \sim \exp \left(\frac{k_{\mathrm{m}}(1)}{3} t\right)-\beta \frac{k_{\mathrm{m}}^{\prime}(1)}{30} \exp \left[k_{\mathrm{m}}(1) t\right]\left[1-\exp \left(-\frac{2 k_{\mathrm{m}}(1)}{3} t\right)\right],
\end{aligned}
$$

where $k_{\mathrm{m}}^{\prime}(c)=\mathrm{d} k_{\mathrm{m}}(c) / \mathrm{d} c$, and $S_{0}$ and $S_{1}$ refer to the $O(1)$ and $O(\beta)$ terms, respectively, in the expansion of $S$. In this regime all the cells have an adequate supply of nutrient; this leads, for the usual reasons, to exponential growth in $S_{0}$, and this is consistent with the study by Greenspan (1972), for example. These expansions breakdown when $t \sim \ln (1 / \beta)$ so that $S_{0}^{2}=O(1 / \beta)$; for $t=\ln (1 / \beta)+O(1)$, a full balance holds in (5.1) and (5.3) under appropriate rescalings. The numerical and the asymptotic solutions for the growth speed on the time scale discussed in this section are included in Fig. 11 for $\varepsilon=0.01$ and $\beta=0.1$, with $m_{1}=m_{2}=5, c_{\mathrm{c}}=c_{\mathrm{d}}=0.5, \delta=0.5$, and $\sigma=0.9$. Figure 11 shows good 


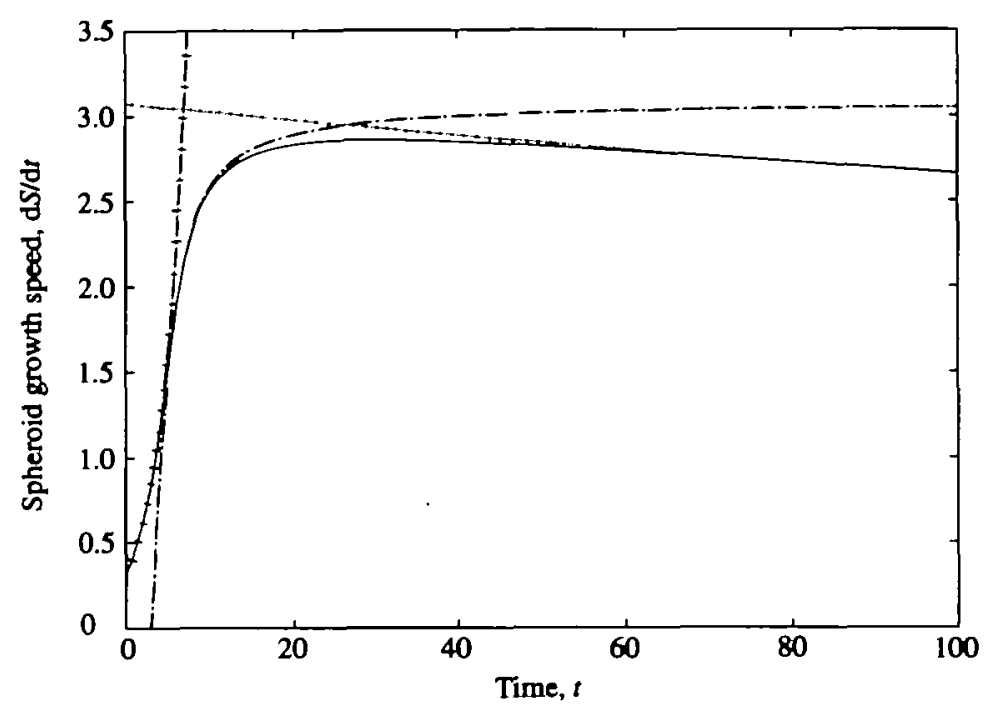

FIG 11 Plots of growth speed against time for $\varepsilon=0.01$ : (-) the numerical solution to the full system and asymptotic approximatıons for $\left(-x_{-}\right)$the early time scale, $(-,-)$the mid time scale, and $(\cdots)$ the long time scale.

agreement with the early time expansion up to about $t=5$, when the central cells cease to proliferate, which slows the growth.

5.2.3 Large-time behaviour. We now discuss the large time behaviour of (5.1-5.3); the analysis is applicable for $t \gg 1$ if $\beta=O(1)$ and for $t-\ln (1 / \beta) \gg 1$ for $\beta \ll 1$. For $(5.1-5.3)$ to be valid as a leading-order balance we also require that $t \ll 1 / \varepsilon$. The results of this section provide matching conditions for the larger time scales discussed below.

Following the early time scale of the previous subsection, the tumour spheroid grows large and the concentration of the nutrient in the core diminishes through consumption, the cells there consequently becoming quiescent. In discussing the equations $(5.1-5.3)$ in the limit $t \rightarrow \infty$ two regions must be analysed, (i) a rim in which $c=O(1)$ and (ii) the central core in which $c \ll 1$. Taking the limit $S \rightarrow \infty$, we seek power-series expansions of $c$ and $v$ in terms of $1 / S$ for each region and then match them together; we shall find that $S \sim q t$ as $t \rightarrow \infty$ for some constant $q$, which is defined below.

(i) The viable rim. To focus on the rim region as $S \rightarrow \infty$ we translate the equations using $z=r-S(t)$ to fix the spheroid surface at $z=0$, the rim being given by $z=O(1)$. Equation (5.1) becomes

$$
\frac{\partial^{2} c}{\partial z^{2}}+\frac{2}{z+S} \frac{\partial c}{\partial z}=\beta \frac{c^{m_{1}}}{c_{\mathrm{c}}^{m_{1}}+c^{m_{1}}} .
$$

We write

$$
c \sim c_{0}(z)+S^{-1}(t) c_{1}(z)
$$

the expansion for $v$ being given in terms of $c$ by (5.2). The fact that $c_{0}$ and $c_{1}$ are 
functions of $z$ only is a consequence of the analysis that follows, but for brevity we shall assume this from the start.

We thus obtain

$$
\frac{\mathrm{d}^{2} c_{0}}{\mathrm{~d} z^{2}}=\beta \frac{c_{0}^{m_{1}}}{c_{\mathrm{c}}^{m}+c_{0}^{m_{1}}}
$$

and using the matching condition that $c_{0} \rightarrow 0$ as $z \rightarrow-\infty$ we obtain

$$
\frac{\mathrm{d} c_{0}}{\mathrm{~d} z}=\left(2 \beta \int_{0}^{c_{0}} \frac{c^{m_{1}}}{c_{\mathrm{c}}^{m_{1}}+c^{m_{1}}} \mathrm{~d} c\right)^{\frac{1}{2}} \equiv h\left(c_{0}\right) .
$$

The integral in (5.6) can be evaluated in closed form for integer $m_{1}$; for example, in the simplest case of $m_{1}=1$ we have

$$
h\left(c_{0}\right)=\left\{2 \beta\left[c_{0}+c_{c} \ln \left(\frac{c_{c}}{c_{0}+c_{c}}\right)\right]\right\}^{\frac{1}{2}} .
$$

Equation (5.6) implies

$$
\int_{c_{0}}^{l} \frac{\mathrm{d} c}{h(c)}=-z
$$

The correction term $c_{1}$ satisfies

$$
\frac{\mathrm{d}^{2} c_{1}}{\mathrm{~d} z^{2}}+2 \frac{\mathrm{d} c_{0}}{\mathrm{~d} z}=\beta k_{\mathrm{m}}^{\prime}\left(c_{0}\right) c_{1}
$$

Hence

$$
\frac{\mathrm{d}}{\mathrm{d} c_{0}}\left(h\left(c_{0}\right)^{2} \frac{\mathrm{d} c_{1}}{\mathrm{~d} c_{0}}-h\left(c_{0}\right) \frac{\mathrm{d} h}{\mathrm{~d} c_{0}} c_{1}\right)=-2 h\left(c_{0}\right),
$$

giving the integral expression for $c_{1}$

$$
c_{1}=2 h\left(c_{0}\right) \int_{c_{0}}^{1} \frac{1}{h(c)^{3}}\left(\int_{0}^{c} h(\psi) \mathrm{d} \psi\right) \mathrm{d} c,
$$

where we have used $h(0)=0$ and $c_{1}=0$ on $z=0$.

It follows from (5.2) that $v(z, t) \sim h\left(c_{0}\right) / \beta$ and, including the first correction term, the growth speed of the tumour is given by

$$
v(0, t)=\frac{\mathrm{d} S}{\mathrm{~d} t} \sim q-\frac{1}{S} \frac{2}{\beta h(1)} \int_{0}^{1} h(c) \mathrm{d} c,
$$

where the constant $q=h(1) / \beta$ is the asymptotic growth speed of the spheroid on this time scale, with $S \sim q t$, and it is readily calculated (at least numerically) for any $m_{1}$ using the definition (5.6). The speed $q$ is therefore approached from below as $t \rightarrow \infty$, and it gives an approximation to the maximum growth speed attained by the spheroid. 
In order to match into the core region discussed below, we need to determine the behaviour as $z \rightarrow-\infty$, where $c_{0} \rightarrow 0$. It is easily shown from (5.6) that

$$
\begin{array}{lll}
c_{0} \sim\left(\frac{\left(m_{1}-1\right)^{2} \beta(-z)^{2}}{2\left(m_{1}+1\right) c_{c}^{m_{1}}}\right)^{-1 /\left(m_{1}-1\right)} & \text { as } z \rightarrow-\infty \text { for } m_{1}>1, \\
c_{0} \sim A_{0} \exp \left[\left(\beta / c_{c}\right)^{\frac{1}{2}} z\right] & \text { as } z \rightarrow-\infty \text { for } m_{1}=1,
\end{array}
$$

where the constant $A_{0}$ can in principle be determined from (5.7).

(ii) The core. The core region is given by $\rho=O(1)$ with $\rho<1$, where we have introduced $\rho=r / S(t)$. In order to match into the rim, it follows from (5.11) that for $m_{1}>1$ we have $c=O\left(S^{-2 /\left(m_{1}-1\right)}\right)$, while $c$ is exponentially small for $m_{1}=1$. It follows from (5.2) that $v=O\left(S^{-\left(m_{1}+1\right) /\left(m_{1}-1\right)}\right)$ for $m_{1}>1$ and is exponentially small for $m_{1}=1$. The velocity in the core is thus small, and it makes a negligible contribution to the speed of tumour growth; this is implicit in (5.10).

In the core we write, for $m_{1}>1$,

$$
c \sim S(t)^{-2 /\left(m_{1}-1\right)} C_{0}(\rho)
$$

giving

$$
\frac{1}{\rho^{2}} \frac{\mathrm{d}}{\mathrm{d} \rho}\left(\rho^{2} \frac{\mathrm{d} C_{0}}{\mathrm{~d} \rho}\right)=\frac{\beta}{c_{\mathrm{c}}^{m_{1}}} C_{0}^{m_{1}}
$$

subject to

$$
\begin{array}{ll}
\text { at } \rho=0 & \frac{\mathrm{d} C_{0}}{\mathrm{~d} \rho}=0, \\
\text { as } \rho \rightarrow 1^{-} & C_{0} \sim\left(\frac{\left(m_{1}-1\right)^{2} \beta(1-\rho)^{2}}{2\left(m_{1}+1\right) c_{c}^{m_{1}}}\right)^{-1 /\left(m_{1}-1\right)},
\end{array}
$$

where we have matched with (5.11). This may be illustrated by the special case $m_{1}=5$ in which it is well known that the Emden-Fowler equation (5.12) can be solved explicitly to give

$$
C_{0}=\left(\frac{3 c_{\mathrm{c}}^{5}}{\beta}\right)^{\frac{1}{4}} \frac{1}{\left(1-\rho^{2}\right)^{\frac{1}{2}}} .
$$

For $m_{1}=1$ the leading-order solution in the core is given by

$$
c \sim \frac{2 A_{0} S(t)}{r} \exp \left[-\left(\beta / c_{c}\right)^{\frac{1}{2}} S(t)\right] \sinh \left[\left(\beta / c_{c}\right)^{\frac{1}{2}} r\right],
$$

where we have again matched with (5.11). Expression (5.13) is, as expected, exponentially small in $S$ and the full balance in (5.13) holds for $r=O(1)$; contrast this with (5.12), which is applicable for $\rho=O(1)$ with $m_{1}>1$. We shall not discuss the case $m_{1}<1$, for which the solution develops a region at the centre of the tumour in which $c$ is identically zero; for large $t$ this encompasses the whole of the core and some of the rim. Nevertheless, the qualitative form of the tumour growth rate is unaffected. 


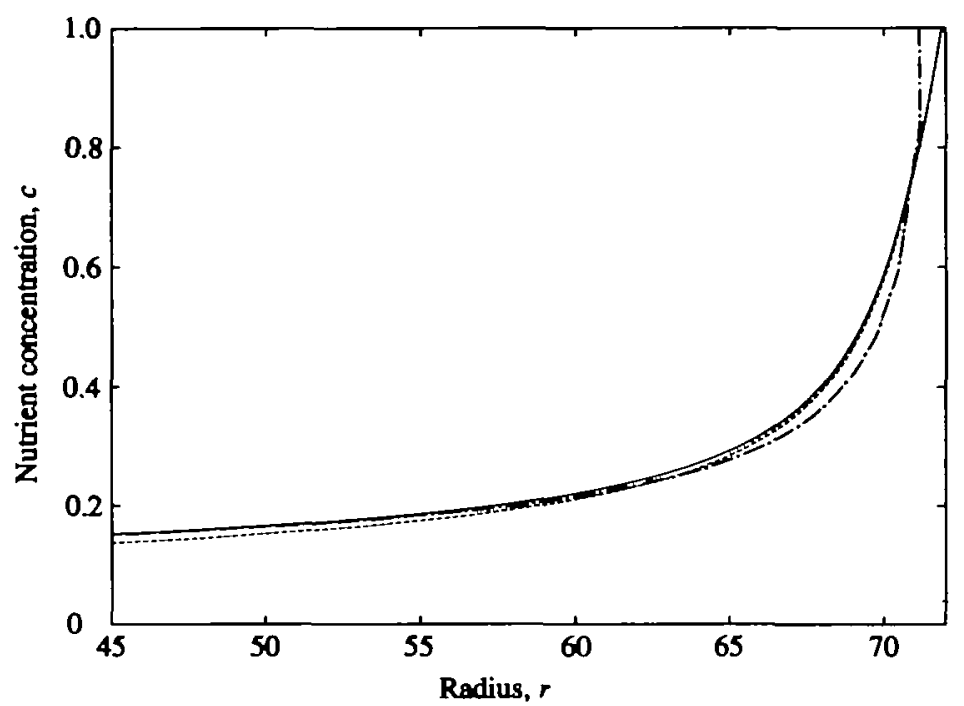

FIG. 12. Plots of the nutrient concentration against the radius for $\varepsilon=0.01$ : ( $\longrightarrow$ numerical solution, $(--)$ asymptotic rim solution, and (- - ) asymptotuc core solution.

The matching of the asymptotic core- and rim-nutrient distributions is demonstrated and compared to the numerical solution in Fig. 12 for $\varepsilon=0.01$ (see Section 5.2.2 for the other parameter values) and $t=30$, giving $S \approx 72$. Although $t$ is approaching $1 / \varepsilon$ (and $n \approx 0.9$ in the core), the asymptotic solutions still agree well with the numerical solution. The growth speed given by (5.10) for this time scale is compared with the results of the same simulation in Fig. 11. Observe that the corresponding curve (dash-dot line) picks up the numerical solution around where the early-time expansion begins to fail. However, the curves then diverge at about $t=15$, where $S \approx 30$, but the numerical solution is picked up by the long-time solution of Section 5.4 (given by the dotted line) at about $t=50$.

There are two further time scales to discuss. On the first time scale $\left(t=O\left(\varepsilon^{\left.-\left(m_{1}-1\right) / 2 m_{1}\right)}\right)\right.$ for $\left.m_{1}>1\right)$ cell death starts to influence the core velocity, while on the second time scale $(t=O(1 / \varepsilon))$ a fully necrotic core develops, the asymptotic spatial structure being of the qualitative form observed in practice.

\section{$5.3 t=O\left(\varepsilon^{-\left(m_{1}-1\right) / 2 m_{1}}\right)$}

The leading-order behaviour in the rim on the first time scale is still determined as in Section 5.2 .3 , so that $S \sim q t$, but, on introducing the scalings

$$
t=\varepsilon^{-\left(m_{1}-1\right) / 2 m_{1}} \hat{t}, \quad r=\varepsilon^{-\left(m_{1}-1\right) / 2 m_{1}} \hat{r}, \quad c=\varepsilon^{1 / m_{1}} \hat{c}, \quad v=\varepsilon^{\left(m_{1}+1\right) / 2 m_{1}} \hat{v},
$$

the leading-order core solution for $m_{1}>1$ is given by

$$
n \sim 1 \text {, }
$$

with

$$
\frac{1}{\hat{r}^{2}} \frac{\partial}{\partial \hat{r}}\left(\hat{r}^{2} \frac{\partial \hat{c}_{0}}{\partial \hat{r}}\right)=\beta \frac{\hat{c}_{0}^{m_{1}}}{c_{c}^{m_{1}}}
$$




$$
\begin{array}{ll}
\text { at } \hat{r}=0 & \frac{\partial \hat{c}_{0}}{\partial \hat{r}}=0, \\
\text { as } \hat{r} \rightarrow q \hat{t}^{-} & \hat{c}_{0} \sim\left[\left(m_{1}-1\right) \beta(q \hat{t}-\hat{r})^{2} / 2\left(m_{1}+1\right) c_{\mathrm{c}}^{m_{1}}\right]^{-1 /\left(m_{1}-1\right)}
\end{array}
$$

(cf. (5.12)), but the leading-order velocity is no longer given by (5.2); rather we have (since $\left.\bar{k}_{\mathrm{d}}(0)=1\right)$

$$
\frac{1}{\hat{r}^{2}} \frac{\partial\left(\hat{r}^{2} \hat{v}_{0}\right)}{\partial \hat{r}}=\frac{\hat{c}_{0}^{m_{1}}}{c_{c}^{m_{1}}}-(1-\delta),
$$

so that

$$
\hat{v}_{0}=\frac{1}{\beta} \frac{\partial \hat{c}_{0}}{\partial \hat{r}}-\frac{1}{3}(1-\delta) \hat{r}
$$

The second term in (5.15) represents the effects of cell death. It follows from (5.14) that $\hat{c}_{0}$ is of the form

$$
\hat{c}_{0}=\hat{t}^{-2 /\left(m_{1}-1\right)} \Phi(\hat{r} / \hat{t}),
$$

so for sufficiently large $\hat{t}$ the velocity (5.15) is negative, despite the fact that most cells are still alive; this is a consequence of cell quiescence and death.

For $m_{1}=1$ similar behaviour occurs, the appropriate rescalings being of the form

$$
t=t_{\mathrm{c}}(\varepsilon) \ln (1 / \varepsilon)+\hat{t}, \quad c=\varepsilon \hat{c}, \quad v=\varepsilon \hat{v},
$$

where $t_{\mathrm{c}}(0)=\left(c_{\mathrm{c}} / \beta q^{2}\right)^{\frac{1}{2}}$, and we obtain for $r=O(1)$

$$
\begin{aligned}
n & \sim 1, \\
\hat{c}_{0} & =\frac{\exp -\left(\beta / c_{\mathrm{c}}\right)^{\frac{1}{2}} q \hat{t}}{r} \sinh \left[\left(\beta / c_{c}\right)^{\frac{1}{2}} r\right],
\end{aligned}
$$

(by a suitable choice of $t_{\mathrm{c}}(\varepsilon)$ ) and

$$
\hat{v}_{0}=\frac{1}{\beta} \frac{\partial \hat{c}_{0}}{\partial r}-\frac{1}{3}(1-\delta) r .
$$

$5.4 t=O\left(\varepsilon^{-1}\right)$

This is the second and final time scale of interest. The scalings appropriate to the core are, for $m_{1}>1$,

$$
t=t^{*} / \varepsilon, \quad r=r^{*} / \varepsilon, \quad c=\varepsilon^{2 /\left(m_{1}-1\right)} c^{*},
$$

and we write

$$
S \sim S_{0}^{*}\left(t^{*}\right) / \varepsilon
$$

Expanding in the core in the form

$$
c^{*} \sim c_{0}^{*}\left(r^{*}, t^{*}\right), \quad n \sim n_{0}\left(r^{*}, t^{*}\right), \quad v \sim v_{0}\left(r^{*}, t^{*}\right),
$$


yields

$$
\begin{aligned}
\frac{\partial n_{0}}{\partial t^{*}}+v_{0} \frac{\partial n_{0}}{\partial r^{*}} & =-n_{0}\left[1-(1-\delta) n_{0}\right] \\
\frac{1}{r^{* 2}} \frac{\partial}{\partial r^{*}}\left(r^{* 2} \frac{\partial c_{0}^{*}}{\partial r^{*}}\right) & =\frac{\beta}{c_{c}^{m_{1}}} c_{0}^{* m_{1}} n_{0} \\
\frac{1}{r^{* 2}} \frac{\partial\left(r^{* 2} v_{0}\right)}{\partial r^{*}} & =-(1-\delta) n_{0}
\end{aligned}
$$

Equation (5.17) implies necrosis, with $n_{0}<1$ now occurring; also note that $n_{0}$ and $v_{0}$ decouple from $c_{0}^{*}$. The problem for $n_{0}$ and $v_{0}$ is independent of $m_{1}$ and remains valid for $m_{1}=1$, when $c$ is exponentially small in the core. The required boundary conditions are

$$
\begin{array}{ll}
\text { at } r^{*}=0 & v_{0}=0, \frac{\partial c_{0}^{*}}{\partial r^{*}}=0, \\
\text { as } r^{*} \rightarrow S_{0}^{*}\left(t^{*}\right) & n_{0} \rightarrow 1, c_{0}^{*} \rightarrow \infty, \\
\text { as } t^{*} \rightarrow 0^{+} & S_{0}^{*} \rightarrow 0 .
\end{array}
$$

The condition on $r^{*}=S_{0}^{*}$ arises from matching into the viable rim, whose structure is as described earlier, so that $S_{0}^{*}$ is determined by

$$
\frac{\mathrm{d} S_{0}^{*}}{\mathrm{~d} t^{*}}=q-Q\left(t^{*}\right)
$$

subject to $S_{0}^{*}(0)=0$. Here $Q\left(t^{*}\right) \equiv-v_{0}\left(S_{0}^{*}\left(t^{*}\right), t^{*}\right)$ is determined by $(5.17-5.20)$ so the second term on the right-hand side of (5.21) is the (negative) contribution to tumour growth due to cell death in the core, while the first term gives the rate of increase in size due to cell proliferation in the viable rim.

On integrating, we obtain from (5.17) and (5.19)

$$
\frac{\partial v_{0}}{\partial t^{*}}-(1-\delta) v_{0} n_{0}=-v_{0}
$$

Using (5.20) we can deduce that $Q\left(t^{*}\right)$ satisfies

$$
\frac{\mathrm{d} Q}{\mathrm{~d} t^{*}}=-\left(\delta+2 \frac{\mathrm{d}}{\mathrm{d} t^{*}}\left(\ln S_{0}^{*}\right)\right) Q+(1-\delta) \frac{\mathrm{d} S_{0}^{*}}{\mathrm{~d} t^{*}}
$$

Therefore, $S_{0}^{*}$ and $Q$ are determined by the system

$$
\begin{aligned}
& \frac{\mathrm{d} S_{0}^{*}}{\mathrm{~d} t^{*}}=q-Q, \\
& \frac{\mathrm{d} Q}{\mathrm{~d} t^{*}}=-\delta Q+\left(1-\delta-\frac{2 Q}{S_{0}^{*}}\right)(q-Q),
\end{aligned}
$$

with $S_{0}^{*}(0)=Q(0)=0$. 
The system (5.24-5.25) has the small-time behaviour

$$
S_{0}^{*} \sim q t^{*}-\frac{1}{6}(1-\delta) q t^{* 2}, \quad Q \sim \frac{1}{3}(1-\delta) q t^{*}-\frac{1}{36}(1-\delta)(2+\delta) q t^{* 2}
$$

as $t^{*} \rightarrow 0$, while the large-time behaviour is

$$
S_{0}^{*} \sim \delta q t^{*}+2(1-\delta) q \ln t^{*}, \quad Q \sim(1-\delta) q-2(1-\delta) q / t^{*}
$$

as $t^{*} \rightarrow \infty$. The reduction of the tumour growth speed $S_{0}^{*}$ from $q$ to $\delta q$ is to be expected; as $t^{*} \rightarrow \infty$, equilibration occurs between the total cell birth rate in the viable rim and the total death rate in the core; the factor $\delta$ corresponds to the loss of volume on cell death. Thus we have $U \sim \delta q$ as $\varepsilon \rightarrow 0$.

In (5.24-5.25), $S_{0}^{*}$ and $Q$ are each proportional to $q$, the only other parameter being $\delta$. Limiting cases of $\delta$ are of some interest; some examples follow.

(i) $0<1-\delta \ll 1$ (small volume loss on cell death). In this case

$$
\begin{aligned}
& S_{0}^{*} \sim q t^{*}-(1-\delta) q\left(t^{*}+2 \int_{0}^{t^{*}}\left(1-\psi-\mathrm{e}^{-\psi}\right) \frac{\mathrm{d} \psi}{\psi^{2}}\right) \\
& Q \sim(1-\delta) q\left[1+2\left(1-t^{*}-\mathrm{e}^{-t^{*}}\right) / t^{* 2}\right]
\end{aligned}
$$

and $n_{0}$ is given to leading order by

$$
n_{0} \sim \mathrm{e}^{-t^{*}+r^{*} / q} .
$$

In Fig. 13 this solution for $n$ (dotted line) is compared with the numerical solutions of

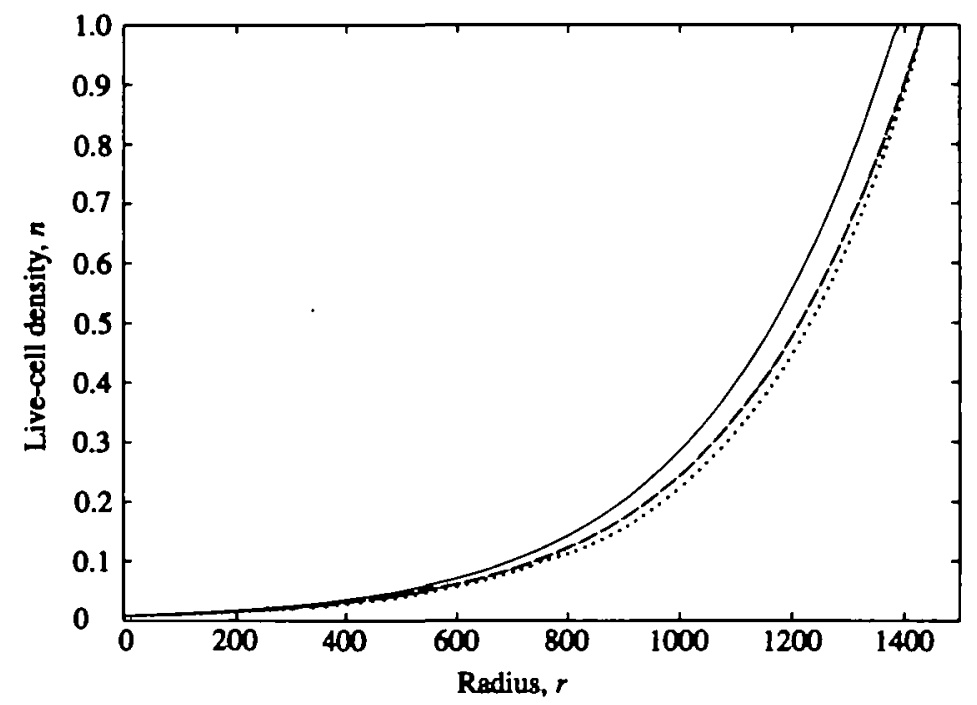

FIG. 13. Plots of the live-cell density against radius at $t=500$, for $\varepsilon=0.01$ and $1-\delta=0.1:(\longrightarrow)$ the numerical solution of the full model, $(-\cdots)$ the numerical solution of the $t=O(1 / \varepsilon)$ model, and $(\cdots)$ small $1-\delta$ solutions. 
the full (solid line) and long-time models (dashed line) and the asymptotic solutions for $\varepsilon=0.01,1-\delta=0.1, m_{1}=m_{2}=1, c_{c}=c_{d}=0.5$, and $\sigma=0.9$ for time $t=500$. The long-time curves are slightly displaced forward due to the difference between $S$ and $S_{0}^{*}$, but there is good agreement between the long-time solution and the asymptotic approximation.

(ii) $\delta=0$. This case corresponds to the case analysed by, for example, Greenspan (1972), whereby it is assumed that (ultimately) cells lose all their volume following death; note that if the time scales of cell death and decomposition are comparable then $k_{d}$ should be interpreted as the rate at which cells die and decompose rather than simply as the death rate. We obtain

$$
S_{0}^{*}=3 q\left(1-\mathrm{e}^{-r^{*} / 3}\right), \quad Q=q\left(1-\mathrm{e}^{-r^{*} / 3}\right),
$$

giving growth saturation with the radius $S_{0}^{*}=3 q$.

(iii) $0<\delta \ll 1$ (large volume loss). For $t^{*}=O(1)$, the solution is given at leading order by (5.28). On the longer time scale $T^{*}=O(1)$, with $T^{*}=\delta t^{*}$,

$$
S_{0}^{*}-2 q \ln \left(S_{0}^{*}\right) \sim q T^{*}+3 q-2 q \ln (3 q), \quad Q \sim q,
$$

which indicates how the large-time growth speed $\delta q$ is established.

Given $S_{0}^{*}$ and $Q,(5.19)$ and (5.22) may easily be solved by characteristic methods to give the solution in terms of a parameter $\tau^{*}$ as

$$
\begin{aligned}
& r^{* 3}=S_{0}^{* 3}\left(\tau^{*}\right)-3 Q\left(\tau^{*}\right) S_{0}^{* 3}\left(\tau^{*}\right)\left(1-\mathrm{e}^{\tau^{*}} \mathrm{e}^{-t^{*}}\right), \\
& \nu_{0}=-Q\left(\tau^{*}\right) S_{0}^{* 2}\left(\tau^{*}\right) \mathrm{e}^{\tau^{*}} \mathrm{e}^{-t^{*}} / r^{* 2}, \\
& n_{0}=\mathrm{e}^{\tau^{*}} \mathrm{e}^{-t^{*}} /\left[\delta+(1-\delta) \mathrm{e}^{\tau^{*}} \mathrm{e}^{-t^{*}}\right] .
\end{aligned}
$$

The most instructive aspect of this solution is the behaviour as $t^{*} \rightarrow \infty$, for which we have (using (5.27))

$$
\begin{aligned}
\delta q \ln \left(\frac{-v_{0}}{(1-\delta) q}\right)- & v_{0}-(1-\delta) q \sim z^{*}, \\
n_{0} & \sim \frac{v_{0}}{(1-\delta)\left(v_{0}-\delta q\right)},
\end{aligned}
$$

where $z^{*}=r^{*}-S_{0}^{*}\left(t^{*}\right)$. The solution thus evolves to a travelling wave of width $z^{*}=O(1)$ where the cells, which have become quiescent in the viable rim, then die. In other words, for large $t^{*}$ we have the following three-layer structure.

(i) Viable rim, $S_{0}^{*}\left(t^{*}\right)-r^{*}=O\left(\varepsilon \beta^{-\frac{1}{2}}\right)$. The behaviour in this region is determined by the availability of nutrient, as described by (5.5). The cells become quiescent within this layer.

(ii) Quiescent layer, $S_{0}^{*}\left(t^{*}\right)-r^{*}=O(1)$. The behaviour is governed by the rate of cell death in the absence of nutrient and is given by (5.29-5.30).

(iii) Necrotic core, $r^{*}=O\left(t^{*}\right), r^{*} / \delta q t^{*}<1$. Here the live cell concentration is exponentially small. 


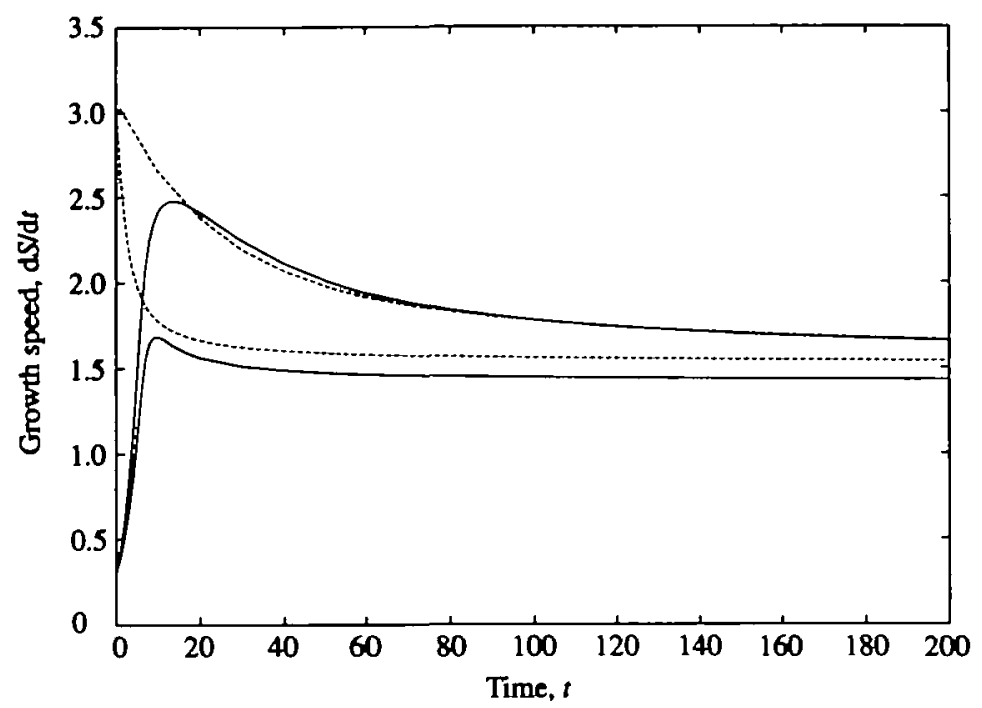

FIG 14. Plots of the growth speed against time showing the numerical solution of $(-)$ the full model and (- - ) the long-time solutions for $\varepsilon=0.1$ (upper curves) and $\varepsilon=1$ (lower curves).

We have thus derived this well-established structure by asymptotic methods as a natural consequence of the model, rather than by a priori assumptions.

Figure 11 gives the growth-speed curve for $\varepsilon=0.01$. The long-time solutions for larger values of $\varepsilon$ are shown in Fig. 14 for $\varepsilon=0.1$ and $\varepsilon=1$; the other parameters are as given in Section 5.2.2. The graph shows very good agreement for the case $\varepsilon=0.1$ as the speed tends to its asymptote $U \approx 1.53$. Even for $\varepsilon=1$ the qualitative agreement is surprisingly good. Note that with these parameter values the case $a(1)=0$, implying $U=0$, occurs when $\varepsilon \approx 7.6$.

\section{Discussion}

Existing mathematical models, such as that of Greenspan (1972), assume that a spheroid is divided into compartments of growing live cells, mitotically inhibited cells, and (contracting) dead cells. These simple models can capture all phases of spheroid growth and provide a means of gleaning information through analytical study, but this is at the cost of imposing a priori a number of assumptions about tumour structure. The tumour-growth model presented in this paper is new in that it treats the tumour spheroid as a continuum of both living and dead cells, with the local volume change from growth and death creating a continuous velocity field within the spheroid. The diffusion and consumption of a generic nutrient are the only mechanisms for driving cell growth and death which are included, and the model succeeds in capturing (without ad hoc assumptions) the initial ('exponential') and the later (linear) phases of growth. This suggests that the diffusion of nutrients is perhaps the most important factor in governing spheroid growth during these phases. Further, fairly good quantitative agreement with the available experimental data can be achieved.

It is well known that the presence of nonproliferating cells in tumours significantly af- 
fects the success of treatment via radiotherapy. This mode of therapy tends to target the proliferating cells; the surviving quiescent cells can thus proliferate afterwards because of the renewed nutrient supply, and the tumour may then recover. The prediction of an inner rim of quiescent cells due to low nutrient levels arises naturally from the model; its existence is due to a time lag of cells dying under these conditions. It was shown in Section 5 that this time delay is responsible for the two phases of growth retardation following the initial exponential phase, the first being due to nutrient-diffusion limitations preventing the core cells from proliferating and then a second retardation occurs due to necrosis. In practice, this type of behaviour will be difficult to observe, since most experiments start with a spheroid consisting of $O\left(10^{5}\right)$ cells; the travelling-wave formulation of Section 4 and the long-time equations of Section 5.4 is then the most appropriate formulation. Nevertheless, it would be interesting to have experimental confirmation of the phenomenon. The analysis of Section 4.2 offers insight into the linear-growth phase of the spheroid, and equation (4.14) provides a useful indication of how deeply quiescent cells extend into a well-developed spheroid. In order for more detailed quantitative comparison to be pursued, there is a need for more experimental data, especially with regard to cell death in nutrient-starved conditions. In particular, precise experimental values for the maximum death-rate parameter, $B$, would be valuable in assessing the validity of the assumptions of the analysis in Section 5 .

There is some interest in obtaining ordinary-differential-equation (or, better still, analytical) formulations for tumour growth, for example, in order to assess by simple means the role of the various physical mechanisms. The asymptotics of Section 5 go some way to deriving such models from physically more realistic partial-differential-equation formulations. Some examples follow.

(i) For $t=O(1)$ and $\beta \ll 1$ the moving-boundary problem (5.1) and (5.3) for a single quasisteady reaction-diffusion equation describes the transition from an exponentialgrowth to a linear-growth phase. This formulation can be further simplified to a single first-order ordinary differential equation in $t$ in some special cases; notably, $m_{1} \gg 1$, and the linear case $m_{1}=1, c_{\mathrm{c}} \gg 1$.

(ii) For $t=O(1 / \varepsilon)$ the formulation reduces to a second-order system of ordinary differential equations (5.24) and (5.25), which can be solved analytically for limiting values of $\delta$. For $\delta>0$ this gives a transition from one growth rate $\dot{S} \sim q$ to a slower one $\dot{S} \sim \delta q$. While for $\delta=0$ it provides a transition from linear growth to saturation; this makes explicit an unphysical assumption that is inherent in the existing approaches to saturation, namely that the material which comprises a cell completely disappears after its death.

Figures 11 and 14, in particular, indicate the applicability of such simplified versions of the model. Note that on the time scale of Section 5.3 the tumour growth is simply linear to leading order.

Finally, note that for $\delta>0$ the current model lacks any mechanism to produce the eventual saturation of spheroid growth. In experiments, it is observed that a viable rim of cells remains during saturation, with a regular doubling time. Although several studies have investigated the effects of environmental factors on the eventual saturation size, there is very little information on what actually causes the saturation of growth. Mitotic inhibitors have been extracted from the necrotic core (see, for example, Levine et al., 1984), and these pre- 
sumably diffuse through the spheroid affecting the mitotic behaviour of some of the cells; however, this mechanism alone cannot be responsible for saturation since it requires that all the cells be inhibited if a continued increase in volume due to cell reproduction is to be prevented; this contradicts experimental observations. During mitosis, the reduced strength in binding between cells may cause individual cells to be shed into the surrounding matrix (Weiss, 1978; Landry et al., 1981) however, the cells that remain will still be reproducing so this mechanism cannot explain growth saturation either. In the models of Greenspan (1972) and subsequent similar studies, the crucial parameter for saturation arises in an expression stating that the contraction rate of the necrotic core is proportional to its volume; it is suggested that this is due to the process of disintegration of necrotic cellular material into simpler permeable compounds with a subsequent loss in volume. However, no indication is given as to what causes the disintegration of the necrotic products or as to what ultimately happens to the chemicals produced. Currently under investigation are possible mechanisms for the volume loss in the necrotic core due to the utilization of necrotic products by neighbouring live cells and through leakage by diffusion into the surrounding matrix. An extension to the mathematical model of this paper can then lead to the prediction of growth saturation, again without making any assumptions on the spheroid structure.

\section{Acknowledgements}

The first author acknowledges the support of an EPSRC Earmarked Research Studentship and the second a Nuffield Foundation Science Research Fellowship. We are very grateful to Dr M. A. J. Chaplain and Professor T. G. Rogers for helpful conversations.

\section{Appendix: Parameter values}

The notation in this appendix reverts to that of the model prior to nondimensionalization. Despite the huge amount of experimental literature available, it is difficult to accurately assess a number of the parameter values required for the model. Cell diameters are in the range 10-100 $\mu \mathrm{m}$ (Melicow, 1982) depending on the cell line, with $V_{\mathrm{L}} \approx 10^{-9} \mathrm{~cm}^{3}$ per cell being typical ( $\mathrm{Li}, 1982 \mathrm{a}$; Casciari et al., 1992a). In the model described by Landry et al. (1982), $V_{\mathrm{D}} \approx 0.5 V_{\mathrm{L}}$ was assumed. The diffusion coefficient of the nutrient will again be dependent on the type of nutrient and cell line; that of glucose has been measured to be $1.1 \times 10^{-6}$ and $4 \times 10^{-7} \mathrm{~cm}^{2} \mathrm{~s}^{-1}$ by Casciari et al. (1988) and by Freyer $\&$ Sutherland (1983), respectively, while that of oxygen is around $2 \times 10^{-5} \mathrm{~cm}^{2} \mathrm{~s}^{-1}$ (Hlatky \& Alpen, 1985). Casciari et al. (1992b) modelled the oxygen consumption as a function of glucose concentration and $\mathrm{pH}$, giving an estimated critical $\left(c_{\mathrm{c}}\right)$ concentration for oxygen of $1.5 \times 10^{-7} \mathrm{~g} \mathrm{~cm}^{-3}$ for EMT6/RO spheroids. Hlatky et al. (1988) investigated (wo cell lines, indicating critical glucose concentrations of $1.4 \times 10^{-4}$ and $1.1 \times 10^{-4} \mathrm{~g} \mathrm{~cm}^{-3}$ for $\mathrm{V} 79$ and 9L cultures, respectively. The value of the external concentration $c_{0}$ depends very much on the proximity to the vasculature of the tumour in vivo or the experimental conditions in vitro.

In terms of the model the rate of growth is given by $k_{\mathrm{m}}(c)-k_{\mathrm{d}}(c)$ and, if the doubling time is $T$ for a concentration $c, k_{m}(c)-k_{\mathrm{d}}(c)=\ln (2) / T$. Li (1982b), plotted the growth rate as a function of glucose concentration revealing a near-step-function relationship. Adopting the step-function forms of $k_{\mathrm{m}}$ and $k_{\mathrm{d}}$ and assuming that the doubling time 
is of $O$ ( 14 hours) under optimal conditions it follows that

$$
A-B(1-\sigma) \approx 10^{-5} \mathrm{~s}^{-1} \text {. }
$$

Even in an optimal nutrient environment, a small number of cells are in practice, observed to die through apoptosis; for example, around 5\% of the surface cells in developed cervical cancers (Moore, 1987) are dead. If the proportion of dead cells is taken to be $\theta \ll 1$, then the live-cell density is approximately $1-\delta \theta$ and, using the assumptions on the long-time behaviour of $n$ above, we have

$$
A \approx B(1-\sigma) / \theta
$$

From this we can deduce that $B(1-\sigma)$ is small compared to $A$ so, from (A.1), $A \approx$ $10^{-5} \mathrm{~s}^{-1}$.

In the papers cited above, the rates of consumption of both glucose and oxygen fit well with the Michaelis-Menten formula $\left(m_{1}=1\right)$, with the critical concentrations for both the growth rate and consumption rate being of a similar order for both glucose and oxygen. This suggests that $\beta k_{\mathrm{m}}(c)+\gamma(c)$ is approximately proportional to $k_{\mathrm{m}}(c)$, so that either $\gamma(c) \equiv 0$ or $\gamma(c) \approx \hat{\gamma} k_{\mathrm{m}}(c)$ for some constant $\hat{\gamma}$. In the latter case we can absorb $\gamma(c)$ into $\beta k_{\mathrm{m}}(c)$ by redefining $\beta$ and setting $\gamma(c) \equiv 0$. Either way the maximum consumption rate is given by $\beta A$. Li (1982a), Hlatky et al. (1988), and Casciari et al. (1992b) all found that, for various cell lines, the maximal consumption rate, $\beta A$, is about $2 \times 10^{-14} \mathrm{gcell}^{-1} \mathrm{~s}^{-1}$ for glucose, and Casciari et al. (1992b) gave $\beta A \approx 2 \times 10^{-15} \mathrm{~g} \mathrm{cell}^{-1} \mathrm{~s}^{-1}$ for oxygen. There currently appears to be no appropriate data available on the remaining parameters $B, c_{\mathrm{d}}$, and $\sigma$. Thus the values adopted in our numerical simulations are estimates.

The parameter values given above are clearly only approximate, and much manipulation of the existing data is required to derive these estimates. Suitable values for the exponents $m_{1}$ and $m_{2}$ are difficult to deduce from the reports of $\mathrm{Li}(1982 \mathrm{a}, \mathrm{b})$, Casciari et al. (1992b), and Hlatky et al. (1988), since Li (1982b) suggested that these exponents are significantly greater than one in the relation of cell growth to glucose concentration, whereas the other reports indicated that the consumption of glucose (as a function of its concentration) fits very well to Michaelis-Menten kinetics, $m_{1}=1$. This discrepancy may be a source of quantitative error in the numerical simulations, although most of the mathematical analysis in this paper requires only that $m_{1}, m_{2} \geqslant 1$.

\section{REFERENCES}

ADAM, J. A., 1986. A simplified mathematical model of tumour growth. Math. Biosct. 81, 224-9.

ADAM, J. A., 1987a. A mathematical model of tumour growth Il: Effects of geometry and symmetry Math. Biosci. 86, 183-211.

ADAM, J. A., 1987b. A mathematical model of tumour growth III: Comparison with experiment. Math. Biosci. 86, 213-27.

ADAM, J. A., \& Maggelakis, S. A., 1989. Mathematical models of tumour growth IV: Effects of necrotic core. Math. Biosci. 97, 121-36.

Burton, A. C., 1966. Rate of growth of solid tumours as a problem of diffusion. Growth, 30, $157-$ 76.

BYRNE, H. M., \& CHAPLAIN, M. A. J.. 1995. Growth of nonnecrotic tumours in the presence and absence of inhibitors. Math. Btosci. 130, 151-81.

BYRNE. H. M., \& CHAPLAIN, M. A. J., 1996. Growth of necrotic tumours in the presence and absence of inhibitors. Math. Biosci., 135, 187-216. 
CARLSSON, J., 1977. A proliferation gradient in three-dimensional colonies of cultured human glioma cells. Int. J. Cancer, 20, 129-36.

CASCIARI, J. J., SOtIRChOS, S. V., \& SUTHERLAND, R. M. 1988. Glucose diffusivity in multicellular tumour spheroids. Cancer Res. 48, 3905-9.

CAsCiARI, J. J., Sotirchos, S. V., \& Sutherland, R. M., 1992a. Mathematical modelling of micro-environment and growth in EMT6/RO multicellular tumour spheroids. Cell Prolif. 25, $1-22$.

Casciari, J. J., Sotirchos, S. V., \& Sutherland, R. M., 1992b. Variation in tumour cell growth rates and metabolism with oxygen-concentration, glucose-concentration and extracellular $\mathbf{p H}$. J. Cell. Physiol. 151, 386-94.

Chaplain, M. A. J., \& BRITTON, N. F., 1993. On the concentration profile of a growth-inhibitory factor in multicell spheroids. Math. Biosci. 115, 233-43.

Chaplain, M. A. J., Benson, D. L., \& MAiNI, P. K., 1994. Non-linear diffusion of a growth inhibitory factor in multicellular spheroids. Math. Biosci. 121, 1-13.

CONGER, A. D., \& ZISKIN, M. C., 1983. Growth of mammalian multicellular tumour spheroids. Cancer Res. 43, 558-60.

COURANT, R., ISAACSON, E., \& REES, M., 1952. On the solution of nonlınear hyperbolic differential equations by fintte differences. Commun. Pure Appl. Math. V, 245-55.

DEAKIN, A. S., 1975. Model for the growth of a solid in vitro tumour. Growth, 39, 159-65.

Folkm AN, J., 1971. Tumour angiogenesis: Therapeutic implications. New Engl. J. Med. 285, 11826.

Folkman, J., \& Hochberg, M., 1973. Self-regulation of growth in three dimensions. J. Exp. Med. $138,745-53$.

FREYER, J. P., 1988. Role of necrosis in regulating the growth saturation of multicell spheroids. Cancer Res. 48, 2432-9.

FREYER, J. P., \& SCHOR, P. L., 1987. Regrowth of cells from multicell tumour spheroids. Cell Tiss. Kinet. 20, 249.

FreYer. J. P., \& SUTHERLAND, R. M., 1983. Determination of apparent diffusion constants for metabolites in multicell tumour spheroids. Adv. Exp. Med. Biol. 159, 463-75.

FREYER, J. P., \& SUTHERLAND, R. M., 1986. Regulation of growth saturation and development of necrosis in EMT6/RO multicellular spheroids glucose and oxygen supply. Cancer Res. 46, $3504-12$.

GLASS, L., 1973. Instability and mitotic pattems in tissue growth. Trans. ASME J. Dyn. Syst. Meas. Cont. G95, 324.

Greenspan, H. P., 1972. Models for the growth of a solid tumour by diffusion. Stud. Appl. Math. $51,317-40$.

HAJI-KARIM, M., \& CARLSSON, J., 1978. Proliferation and viability in cellular spheroids of human origin. Cancer Res. 38, 1457-64.

HLATKY, L., \& ALPEN, E. L., 1985. Two-dimensional diffusion limited system for cell growth. Cell Tiss. Kinet. 18, 597-611

HLATKY, L., SUCHS, R. K., \& ALPEN, E. L., 1988. Joint oxygen-glucose deprivation as the cause of necrosis in a tumour analogue. J. Cell Phys. 134, 167-78.

INCH. W. R., MCCREDIE, J. A., \& SUTHERLAND, R. M., 1970. Growth of modular carcinomas in rodents compared with multicell spheroids in tissue culture. Growth 34, 271-82.

Koch, C. J., KRUUV, J., \& FREY, H. E., 1973. The effect of hypoxia on the generation time of mammalian cells. Radiat. Res. 53, 43-8.

LANDRY, J.. Freyer, J. P., \& SUTHERLAND, R. M., 1981. Shedding of mitotic cells from the surface of multicell spheroids during growth. J. Cell Physiol. 106, 23-32.

LANDRY, J., FreYer, J. P., \& SUTHERLAND, R. M., 1982. A model for the growth of multicell spheriods. Cell Tiss. Kinet. 15, 585-94.

Levine. A. E.. Hamilton, D. A., Yeoman, L. C., BusCh, H., \& Brattain, M. G., 1984. Identification of a tumour inhibitory factor in rat ascites fluid. Biochem. Biophys. Res. Comm. 119, $76-82$

LI. C. K. N., 1982a. The glucose distribution in 9L rat brain multicell tumour spheroids and its effect on cell necrosis Cancer, 50, 2066-73. 
LI, C. K. N., 1982b. The role of glucose on the growth of $9 \mathrm{~L}$ multicell tumour spheroids. Cancer, 50, 2074-8.

LIN, S. H., 1976. Oxygen diffusion in a spheroid cell with non-linear uptake kinetics. J. Theor. Biol. $60,449-57$.

MAGGELAKIS, S. A., 1992. Effects of non-uniform inhibitor production on the growth of cancer cell cultures. Appl. Math. Lett. 5, 11-4.

MAGGELAKIS, S. A., \& ADAM, J.A., 1990. Mathematical model of prevascular growth of a spheroid carcinoma. Math. Comput. Mod. 13, 23-38.

MCELwAIN, D. L. S., 1978. A reexamination of oxygen diffusion in a spheroid cell with MichaelisMentor oxygen uptake kinetics. J. Theor. Biol. 71, 255-67.

MCELwaIN, D. L. S., \& MORRIS, L. E., 1978. Apoptosis as a volume loss mechanism in mathematical models of solid tumour growth. Math. Biosci. 39, 147-57.

MCElwain, D. L. S., \& PETTET, G. J., 1993. Cell migration in multicell spheroids: Swimming against the tide. Bull. Math. Biol. 55, 655-74.

MCELwain, D. L. S., \& Ponzo, P. J., 1977. A model for the growth of a solid tumour with nonuniform oxygen consumption. Math Biosci. 35, 267-79.

MELICOW, M. M., 1982. The three-steps to cancer. A new concept of carcinogenesis. J. Theor. Biol. 94, 471-511.

MoORE, J. V., 1987. Death of cells and necrosis in tumours. In: Perspectives in Mammalian Cell Death (C. S. Potten, ed.). Oxford University Press.

MUELlER-KLIESER, W. F., \& SUTHERLAND, R. M., 1982. Oxygen tension in multicell spheroids of two cell lines. Br. J. Cancer, 45, 256-64.

MUELLER-KLIESER, W. F., FREYER, J. P., \& SUTHERLAND, R.M. 1983. Evidence for a major role of glucose in controlling development of necrosis in EMT6/RO multicell tumour spheroids. Adv. Exp. Med. Biol. 159, 487-95.

Mueller-KLieser, W. F., FREYer, J. P., \& SUTHERLAND, R. M., 1985. Influence of glucose and oxygen supply conditions on the oxygenation of multicellular tumour spheroids. Br. J. Cancer, $53,345-53$.

ShYMKo, R. M., \& GlaSs, L., 1976. Cellular and geometric control of tissue growth and mitotic instability. J. Theor. Biol. 63, 355-74.

ThOMLINSON, R. H., \& GRAY, L. H., 1955. Histological structure of some human lung cancers and the possible implications for radiotherapy. Br. J. Cancer, 9, 539-49.

VAUPEL, P. W., FRINAK, S., \& BICHER, H. I., 1981. Heterogenous oxygen partial pressure and pH distribution in C3H mouse mammary adenocarcinoma. Cancer Res. 41, 2008-13.

WEISS, L., 1978. Some mechanisms involved in cancer cell detachment by necrotic material. Int. J. Cancer, 22, 196-203. 